\title{
Nobel Lecture: LIGO and gravitational waves II
}

\author{
Barry C. Barish \\ California Institute of Technology, Pasadena, California 91125, USA \\ (published 18 December 2018) \\ DOI: 10.1103/RevModPhys.90.040502
}

\section{CONTENTS}

I. Introduction

II. The LIGO Laboratory

III. The LIGO Scientific Collaboration (LSC)

IV. Initial LIGO Performance and Science

V. Advanced LIGO

VI. The Black Hole Merger Event (GW150914)

VII. More Black Hole Mergers

VIII. Science Implications of the Observed

Black Hole Mergers

IX. Virgo Detects Gravitational Waves

X. Neutron Star Binary Merger

XI. Future Directions for LIGO and Gravitational Waves

XII. The Future

XIII. Final Remarks and Reflections

XIV. LIGO_-Some Key Members

Acknowledgments

References

\section{INTRODUCTION}

The observation of gravitational waves (see Fig. 1) in the Laser Interferometer Gravitational-wave Observatory (LIGO) was announced on 11 February 2016 (Abbott et al., 2016a), 100 years after Einstein proposed the existence of gravitational waves (Einstein, 1916, 1918). This observation came after more than 50 years of experimental efforts to develop sensitive enough detectors to observe the tiny distortions in spacetime from gravitational waves. The Nobel Prize for 2017 was awarded to Rainer ("Rai") Weiss, Kip Thorne and myself for decisive contributions to the LIGO detector and the observation of gravitational waves. In fact, the success of LIGO follows from decades of R\&D on the concept and techniques, which were covered in Rai Weiss' Nobel Lecture, followed by the design, construction and evolving the LIGO large-scale interferometers to be more and more sensitive to gravitational waves. This work has been carried through the LIGO Laboratory and the scientific exploitation through the LIGO Scientific Collaboration, having more than 1000 scientists, who author the gravitational wave observational papers. In addition, many others made important contributions to the science of black holes, numerical relativity, etc.

In these three lectures, Rai, Kip and I tell the story of LIGO and gravitational waves in three parts. Rai covers the physics

*The 2017 Nobel Prize for Physics was shared by Rainer Weiss, Barry C. Barish, and Kip S. Thorne. These papers are the text of the address given in conjunction with the award. of gravitational waves, the experimental challenges and some of the pioneering interferometer work. He highlights the experimental challenges and some important early innovations that were proposed, tested at small scale and have been incorporated in the LIGO interferometers. In this lecture, I describe the LIGO project and the improvements that led to detection of merging black holes in Advanced LIGO. I also describe some key features of the interferometers, some implications of the discoveries, and finally, how we envision the evolution of LIGO over the coming decade. Kip will talk

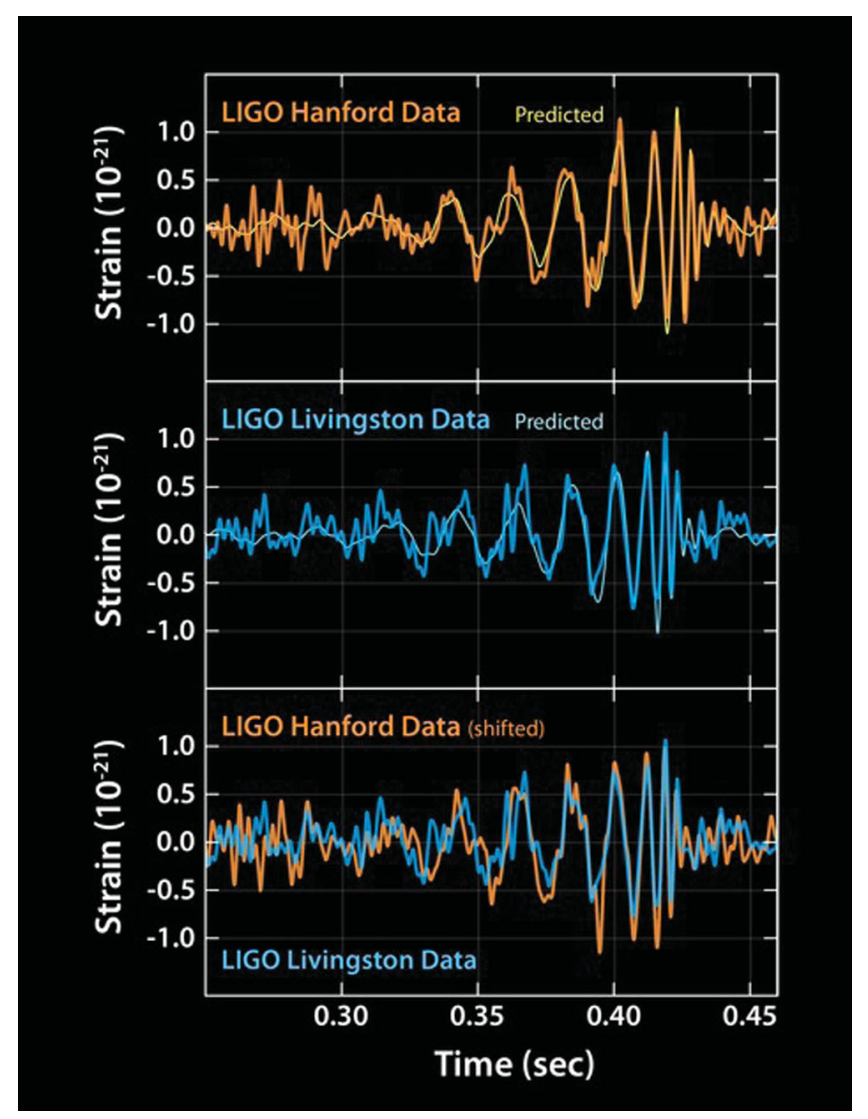

FIG. 1. Figure 1 is the gravitational wave discovery figure that was observed by members of the LIGO Scientific Collaboration within minutes after the event was recorded in Advanced LIGO. Each of the three figures shows the detected "strain" signals in units of $10^{-21}$ vs time. The top trace is the observed waveform detected in the Hanford, Washington interferometer, and the middle trace is the observed waveform in Livingston, Louisiana. The two signals are almost identical, but are shifted by $6.9 \mathrm{msec}$ and are superposed in the bottom trace. From the Caltech/MIT/LIGO Lab. 
about some early personal history, theoretical advances that were crucial to making and interpreting the LIGO, and finally, his vision of the future opportunities in this new field of science.

Our three talks can be read as a series having some overlaps, or can each be read individually. In our lectures, we single out some individuals who played key roles in the discovery of gravitational waves, but by necessity, we have left out many other very important contributors.

\section{THE LIGO LABORATORY}

I became Principal Investigator of LIGO in early 1994 and, consequently, my part of our story begins at that point. A few months earlier, I had become "available," due to the unfortunate cancellation of the Superconducting Super Collider (SSC) in Texas by the U.S. Congress (Riordan, Hoddeson, and Kolb, 2015). The SSC was the realization of a long process by the U.S. and world particle physics communities, conceptually and technically, to develop a facility that would open particle physics to a new energy regime, where there were strong arguments that science beyond the standard model of particle physics would become accessible. The Higgs phenomenon had been proposed to explain the origin of mass for the elementary particles, and the search for the associated Higgs particle was to be the first major goal of the SSC. It was eventually discovered at the Large Hadron Collider at the European Organization for Nuclear Research (CERN) Laboratory in Geneva, Switzerland that has some of the capability of the SSC.

The SSC was designed to have two complementary detectors with different features that would complement each other and confirm discoveries. I was co-spokesperson with Bill Willis of Columbia University for the Gammas, Electronics, Muons (GEM) detector, one of two large detector facilities. We had spent several years in the early 1990s developing concepts, technologies and design for GEM, and in 1993, the conceptual design for GEM had been successfully reviewed. We were just embarking on the final technical design and preparing for the beginning of construction when the SSC was canceled by Congress. This was obviously extremely disruptive to all involved in the SSC. From GEM, a significant contingent of the physicists joined the CERN Large Hadron Collider experiments, and many of our technical developments were incorporated in the CMS and Atlas detectors at CERN.

I had decided not to join the CERN experiments, at least not right away, because I preferred to take my time to decide what I wanted to do next. In fact, I was extremely busy on all the tasks involved in closing down the GEM facilities, and especially, in helping many colleagues I had hired to the SSC find jobs. However, I was approached by the California Institute of Technology (Caltech) to become the LIGO Principal Investigator in early winter 1994. My previous roles on LIGO had been advisory, as I had been a strong supporter for developing the experimental gravitational wave effort at Caltech. I agreed to take over the LIGO leadership, and my first task was to strengthen the LIGO team and to evaluate and revise the LIGO proposal to the National Science Foundation (NSF). The NSF was on a tight schedule to make a decision whether to move forward with LIGO. They wanted an external review of the revised proposal by early summer 1994 and a discussion at the National Science Board later in the summer. They needed a decision whether to go forward with the LIGO construction project by fall.

To organize the LIGO effort, I created a new structure for the project built around the LIGO Laboratory that unified the Caltech and Massachusetts Institute of Technology (MIT) efforts. We divided responsibilities and created an overall concept for developing the two distant instruments in Hanford, Washington and Livingston, Louisiana. The decision to build LIGO at those two sites had already been made, following an evaluation by the NSF of about 20 proposals.

After becoming LIGO PI, my first priority was to make some key hires to strengthen the project, especially from the SSC where some extremely talented individuals had become available. Most importantly, I hired Gary Sanders as project manager, the same position he had with me for GEM at the SSC. Together, we rapidly filled out key positions on LIGO with other senior members, including Albert Lazzarini as integration manager, John Worden for the large vacuum system, Jay Heefner (now deceased) and Rolf Bork to develop digital controls, Dennis Coyne as chief engineer, and others to fill out the initial team.

The next task was to integrate the new members of LIGO with the very talented existing LIGO staff members that included Stan Whitcomb, Robbie Vogt, Bill Althouse, Mike Zucker, Fred Raab at Caltech, and the MIT group under Rai's leadership that included David Shoemaker and Peter Fritschel. The combined Caltech-MIT effort became the LIGO Laboratory, which was to be the organization responsible for the construction and operation of LIGO. LIGO Laboratory is jointly operated by Caltech and MIT through a Cooperative Agreement between Caltech and NSF. LIGO Laboratory includes LIGO Hanford and Livingston Observatories, Caltech and the MIT LIGO facilities. There are currently 178 staff: scientific (including academic staff, postdocs, grad students, engineers, and technicians), and administrative support staff. I stepped down from being LIGO Director in 2006 and Jay Marx became the second LIGO Lab Director. Jay very capably led further improvements of initial LIGO to design sensitivity, as well as the early developments of Advanced LIGO. In 2011, Dave Reitze became LIGO Executive Director and has done a superb job of leading LIGO through the construction and commissioning of Advanced LIGO, and most importantly, the first gravitational wave detections.

In early 1994, we rapidly revised and re-costed the LIGO construction proposal to NSF to account for the larger team, increasing the planned staffing for the Hanford and Louisiana sites, and incorporating a more ambitious technical infrastructure. The plan was to make the initial implementation more robust, as much as possible to be able to accommodate building a second improved version (Advanced LIGO) within the same infrastructure. The total cost of the increases amounted to about $\$ 100 \mathrm{M}$, bringing the total Initial LIGO construction costs to almost $\$ 300 \mathrm{M}$. At the time, this corresponded to our asking for funding for the largest project the NSF had ever undertaken. 
The NSF conducted the external review of our revised proposal in late spring 1994 and we received a very encouraging and strongly positive review. Before making a decision, however, Kip Thorne and I were invited to present LIGO to the National Science Board (NSB). This was very unusual at the NSF, as the NSB does not normally interact directly with proponents. At the NSB meeting, Kip presented the theoretical underpinnings of gravitational waves, as well as giving a description of the physics we would be able to produce with detections. I presented our plans for the project, which involved first building and exploiting Initial LIGO, which would be based as much as possible on technologies we had already demonstrated. This approach was hardly conservative, because LIGO was such a huge extrapolation from the R\&D prototypes. It was a factor of a 100 in size and at least as large a factor in technical performance. A key feature of our plan was to initiate an ambitious R\&D program to develop and test Advanced LIGO technologies, immediately following the completion of Initial LIGO construction. This would be carried out by keeping the key technical staff that had developed Initial LIGO. This was an unusual request, because Advanced LIGO was only a strategic concept and had not been proposed at this stage. Following our presentations to the NSB, we received formal approval at the full requested funding for Initial LIGO, with a commitment to support the crucial R\&D program for Advanced LIGO.

The basic scheme for LIGO was to use a special high power stabilized single-line laser (neodymium-doped yttrium aluminum garnet $=\mathrm{Nd}$ :YAG) that entered the interferometer and was split into two beams transported in perpendicular directions. The LIGO vacuum pipe is $1.2 \mathrm{~m}$ in diameter and is kept at high vacuum $\left(10^{-9}\right.$ torr $)$. The "test" masses are very highquality mirrors that are suspended, in order to keep them isolated from the Earth. They are made of fused silica and hung in a four-stage pendulum for Advanced LIGO. In the simplest version of the interferometer, the equal length arms are adjusted such that the reflected light from mirrors at the far ends arrives back at the same time, and inverting one, the two beams cancel each other and no light is recorded in the photodetector. This is the normal state of the interferometer working at the "dark port." Many effects make the beams not completely cancel, and the actual optical configuration is more sophisticated.

When a gravitational wave crosses the interferometer, it stretches one arm and compresses the other, at the frequency of the gravitational wave. Consequently, the light from the two arms returns at slightly different times (or phase) and the two beams no longer completely cancel. This process reverses itself, stretching the other arm and squeezing the initial arm at the frequency of the gravitational wave. The resulting frequency and time-dependent amount of light is recorded by a photosensor and recorded as the waveform from the passage of a gravitational wave. The experimental challenge is to make the interferometer sensitive to the incredibly tiny distortions of spacetime that come from a gravitational wave, while at the same time, suppressing the various background noise sources.

The spacetime distortions from the passage of an astrophysical source are expected to be of the order of $h=\Delta L / L \sim$ $10^{-21}$, a difference in length of a small fraction of the size of a proton. In LIGO, we have made the length of the interferometer arms as long as is practical, in our case $4 \mathrm{~km}$, and this results in a difference in length we must be sensitive to that is still incredibly small, about $10^{-18} \mathrm{~m}$. For reference, that is about 1000 times smaller than the size of a proton! If that sounds very hard, it is!! Skipping the details, what enables us to achieve this precision is the sophisticated instrumentation that reduces seismic and thermal noise sources, effectively making the statistics very high by having many photons traversing the interferometer arms.

The initial version of LIGO was constructed during the period from 1994 to 1999, employing technologies that represented a balance between being capable of achieving sensitivity levels where the detections of gravitational waves might be "possible," and using techniques that we had fully demonstrated in our laboratories. LIGO was a huge extrapolation from the $30 \mathrm{~m}$ prototype interferometer (Shoemaker et al., 1988) in Garching, Germany and the $40 \mathrm{~m}$ prototype (Abramovici et al., 1996) at Caltech interferometers that preceded it, and especially considering the very large NSF investment, we needed to be confident of technical success. In reality, from the best theoretical estimates at the time, we anticipated that we would likely need to achieve sensitivities well beyond those of Initial LIGO before achieving detections. So, developing the techniques and building Advanced LIGO was always an integral feature of our plans.

\section{THE LIGO SCIENTIFIC COLLABORATION (LSC)}

LIGO Laboratory, even after the strengthening in 1994, was relatively small for building such a large, ambitious and challenging construction project. The key hires that were added to the original Caltech and MIT LIGO teams were focused in areas needed for the construction of this sophisticated and challenging project. There were other crucial areas where we were weak. These were basically in areas that would be needed to extract the science, building computing facilities, data analysis infrastructure, search algorithms and pipelines, etc. In addition, there was expertise in hardware areas outside Caltech and MIT that could be strengthened by involving expertise from the larger worldwide community.

Gary Sanders and I both had worked within high energy physics collaborations. We appreciated the value of such collaborations, but believed LIGO needed a different model. By 1997, in the middle of LIGO construction I made a proposal to the NSF through a review committee chaired by Boyce McDaniel of Cornell, who knew how high energy collaborations worked. Our model for LIGO was somewhat different. We wanted to create a collaboration for LIGO that was focused on the science, an "open" collaboration where individuals or groups could join if they could make significant contributions to LIGO science. They would not be expected to necessarily provide resources or hardware, as is done in high energy physics collaborations. In order to make joining the LSC as attractive as possible, we took a further step to insure that Caltech and MIT LIGO Laboratory scientists would not have a strong built-in advantage to do LIGO science. The step was to make LIGO Laboratory individual scientists join the LSC to do their science and that the science component of 
LIGO would be carried out through that organization. This concept was endorsed by the McDaniel committee, the NSF approved, and we initiated a fledgling collaboration.

It took some effort, but I managed to convince the Caltech and MIT administrations and LIGO Laboratory scientific staff of this plan. I asked Rai Weiss to become the first spokesperson of the LSC and his credibility and approach helped to get the collaboration off the ground. Rai was succeeded by Peter Saulson of Syracuse, who also had a long history in LIGO, was much respected, and was the first spokesperson from outside Caltech and MIT. He was subsequently followed by David Reitze (now LIGO Laboratory Executive Director), Gabriela Gonzalez of Louisiana State University (LSU) and now David Shoemaker (MIT). As the LSC matured, it has become more democratic in involving collaborators, publication policies, etc.

The LSC has all the responsibilities to produce the science with calibrated strain data. The data pipelines have been developed through the LSC and the various analysis groups on different science are organized and run by the LSC. LSC members also participate in some hardware detector areas and future detector R\&D and planning. Also, LSC members participate in calibration of the data and have played a major role in detector characterization of the data. We have a program for young scientists to spend time at the LIGO sites, a "fellows" program.

The LSC has been extremely successful, the proof being the strong and effective role it played in making the discovery of gravitational waves, analyzing the data, interpreting the results, and in writing up and presenting the results. The strong and effective LSC role has continued to the present and the announcement of detection of a neutron star binary merger and all the follow-up measurements with various astronomical instruments.

The LIGO Scientific Collaboration and its 1200 members deserve shared credit for the discoveries reported here, resulting in the Nobel Prize, which must be given to no more than three individuals. The LSC has grown to more than 1200+ scientific collaborators, from 108 institutions and 18 countries! We conducted a study last year to figure out how to evolve the LSC for the gravitational wave observational era we are entering. We expect to institute some changes by the time of the next data run schedule to begin about the end of this calendar year, and we fully expect the LSC to play the central role in producing the future science with LIGO.

\section{INITIAL LIGO PERFORMANCE AND SCIENCE}

We had a two-step concept for LIGO, since beginning the project. Initial LIGO (Abbott et al., 2009), as much as possible, was based on demonstrated methods and technologies, while the second stage, Advanced LIGO, was to achieve significantly improved sensitivities, through implementing methods and technologies that we would develop through an ambitious post construction R\&D program. It was from that perspective that we proposed to the NSF that while we would be commissioning, running and learning from Initial LIGO, we would be funded to simultaneously carry out an ambitious R\&D program to develop the techniques that would improve LIGO in a second step to a sensitivity where detections would become "probable." The NSF approved that plan and funded what was to become a successful R\&D program that began in about 2000 and led to the Advanced LIGO concept that was proposed and approved by the NSF in 2003. The actual project funding was awarded several years later. I emphasize that our being able to carry out this R\&D and design program, keeping key individuals who developed and built Initial LIGO, was crucial to our eventual success in detecting gravitational waves with Advanced LIGO. Another important point is that the Initial LIGO infrastructure was designed such that the interferometer subsystems could be evolved or replaced inside the same infrastructure (vacuum vessels).

After the completion of construction of Initial LIGO (see Fig. 2), we began commissioning and rapidly achieved better sensitivity than any previous gravitational wave detectors. As a result, we embarked on our first gravitational wave search data run. We did not detect gravitational waves, but we did set new astronomical limits on a variety of possible gravitational wave sources. Following the first data run, we made some technical improvements that reduced the background noise levels, both planned improvements and others from what we had learned in the first data run, and then we embarked on a second data run. Again, we did not detect gravitational waves, and again we achieved new limits and published them on various possible sources. All together, we repeated this basic cycle for over a decade, improving sensitivity and taking data for a total of six data runs at ever-increasing sensitivity (see Fig. 3). For the final data runs, the interferometer sensitivities achieved very close to our original Initial LIGO design goals.

We searched for gravitational waves from several potential sources: mergers of binary black holes, a black hole and a
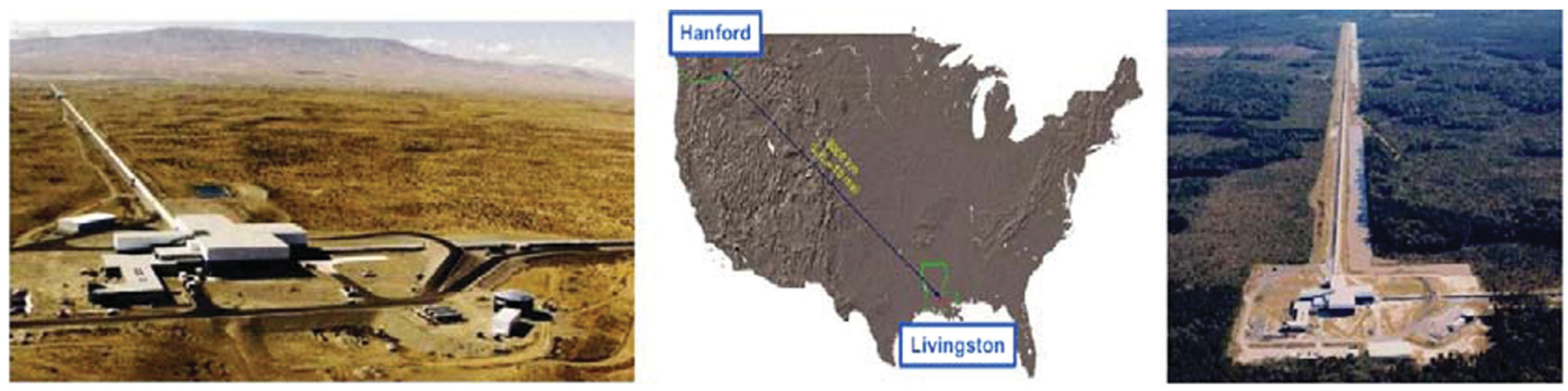

FIG. 2. The LIGO Interferometers in Hanford, Washington and Livingston, Louisiana. From the Caltech/MIT/LIGO Lab. 


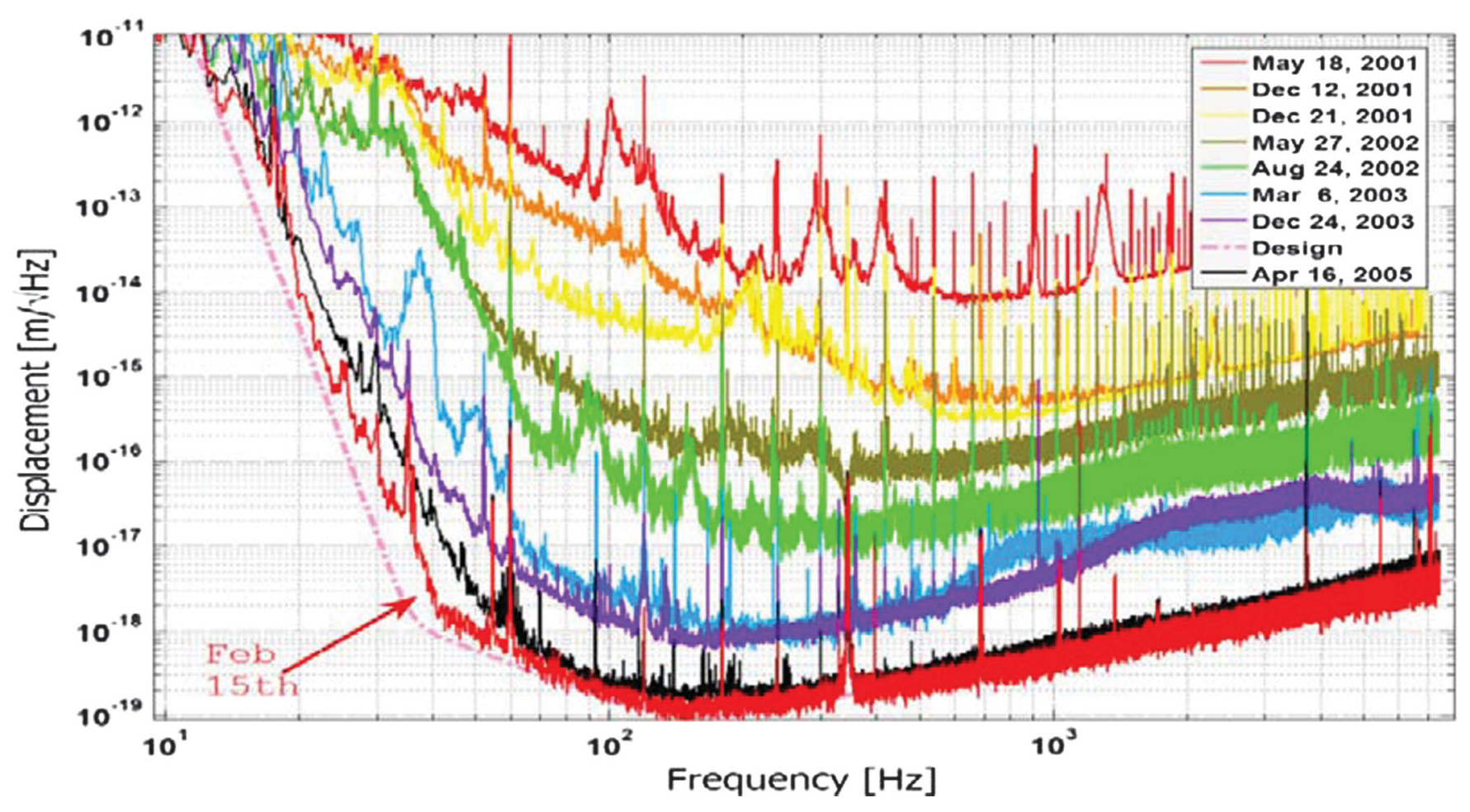

FIG. 3. The evolving improved sensitivity of Initial LIGO.

neutron star, and binary neutron star systems. We also searched for signals from continuous sources, such as known and unknown pulsars, possible stochastic background signals, and unmodeled signals from some new source we had not specifically targeted. Unfortunately, even with the impressive improvements in interferometer sensitivity, we did not detect gravitational waves. The resulting limits on various sources of gravitational waves were constraining for some of the published models of gravitational wave production from astrophysical phenomena.

The final Initial LIGO searches for black hole binary systems were performed in collaboration with the Virgo detector (Aasi et al., 2013). Although we had not detected signals, we were cautiously confident that the technical improvements envisioned for Advanced LIGO would be sufficient to finally achieve detection.

\section{ADVANCED LIGO}

By about 2004, the improved technologies developed for Advanced LIGO (Advanced LIGO, 2015) were mature enough to propose Advanced LIGO to the National Science Foundation. After reviewing the proposal, the NSF continued to support the technical developments in parallel with the continued running of Initial LIGO. The Advanced LIGO project received major funding through the NSF Major Research Equipment and Construction (MREFC), leading us to end the Initial LIGO scientific program and begin construction of Advanced LIGO. Additional significant contributions to Advanced LIGO included a prestabilized laser system from the Max Planck Institute (Germany), test mass suspension systems from the Science and Technology Facilities Council (UK), and thermal compensation wavefront sensors and interferometer control components from the Australian Research Council.

The basic goal of Advanced LIGO is to improve the sensitivity from Initial LIGO by at least a factor of 10 over the entire frequency range of the interferometer (see Fig. 4). It is important to note that a factor of $\mathrm{x} 10$ improvement in sensitivity increases the distance we can search by that factor, since we measure an amplitude. It thus increases the volume of the Universe (or rate for most sources) searched for by a factor of $x 1000$. (The sensitivity to most sources is proportional to the volume we search.) Therefore, there is a very high premium in LIGO on increasing the range we can search, and consequently, we spend a good fraction of our time improving the sensitivity, rather than taking very long data runs.

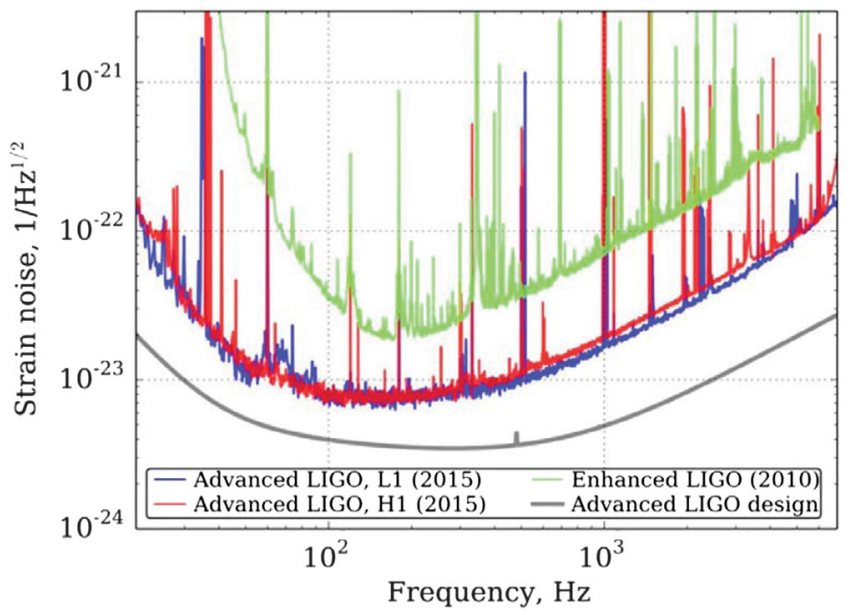

FIG. 4. Advanced LIGO sensitivity goal. 
The Initial and Advanced LIGO gravitational wave detectors are Michelson interferometers with $4 \mathrm{~km}$ long arms. Both use Fabry-Perot cavities to increase the interaction time with a gravitational wave, and power recycling to increase the effective laser power. Signal recycling at the output (dark) port is a new feature of Advanced LIGO, and this changes the control and readout systems. Signal recycling enables tuning the sensitivity response to the physics goals, presently black hole and neutron star mergers. For Advanced LIGO, the design sensitivity is moved to lower frequencies (e.g. $40 \mathrm{~Hz}$ down to $10 \mathrm{~Hz}$ ).

The improved seismic isolation system uses both passive and active isolation, and the improved test mass suspensions use a quadruple pendulum. Higher laser power, larger test masses and improved mirror coatings have been incorporated. The Advanced LIGO interferometers are installed in the same infrastructure, including the same vacuum system as used for Initial LIGO.

The Advanced LIGO laser is a multistage Nd:YAG laser. Our goal is to raise the power from $\sim 18 \mathrm{~W}$ in Initial LIGO to $180 \mathrm{~W}$ for Advanced LIGO, improving the high frequency sensitivity, accordingly. The prestabilized laser system consists of the laser and a control system to stabilize the laser in frequency, beam direction, and intensity. For the results presented here, due to stability issues, heating and scatteredlight effects, the laser power has only modestly been increased. We plan to bring the power up systematically in steps, studying these effects for the next data runs.

The key improvement in Advanced LIGO that enabled the detection of the black hole merger was implementing active seismic isolation and a quadruple suspension system (see Fig. 5).

The multiple suspension system moved all active components off the final test masses and gives better isolation. Initial LIGO used $25-\mathrm{cm}, 11-\mathrm{kg}$, fused-silica test masses, while for Advanced LIGO the test masses are $34 \mathrm{~cm}$ in diameter to reduce thermal noise contributions and are $40 \mathrm{~kg}$, which reduces the radiation pressure noise to a level comparable to the suspension thermal noise. The test mass is suspended by fused-silica fibers, rather than the steel wires used in initial LIGO. The complete suspension system has four pendulum stages, increasing the seismic isolation and providing multiple points for actuation.

The active seismic isolation senses motion and is combined with the passive seismic isolation using servo techniques to improve the low frequency sensitivity by a factor of $\mathrm{x} 100$. Since the rate for gravitational events scales with the volume, this improvement increases the rate for events by $10^{6}$. This improvement enabled Advanced LIGO to make a detection of a black hole merger in days, while Initial LIGO failed to detect gravitational waves in years of data taking.

\section{THE BLACK HOLE MERGER EVENT (GW150914)}

The observation of the first black hole merger by Advanced LIGO (Abbott et al., 2016b, 2016c, 2016d, 2016e) was made on 14 September2015. Figure 1 shows the data, and Fig. 6 reveals the key features of the observed compact binary merger. These are a result of the analysis of the observed event shown in Fig. 1. At the top of Fig. 6, the three phases of the coalescence (inspiral, merger, and ringdown) are indicated above the waveforms. As the objects inspiral together, more and more gravitational waves are emitted and the frequency and amplitude of the signal increases (the characteristic chirp signal). This is following by the final merger, and then, the merged single object rings down. The bottom pane shows on the left scale that the objects are highly relativistic and are moving at more than 0.5 the speed of light by the time of the final coalescence. On the right side, the scale is units of Schwarzschild radii and indicates that the objects are very compact, only a few hundred kilometers apart when they enter our frequency band.

By comparing and fitting our waveform to general relativity, we have concluded that we have observed the merger of

(a)

(b)

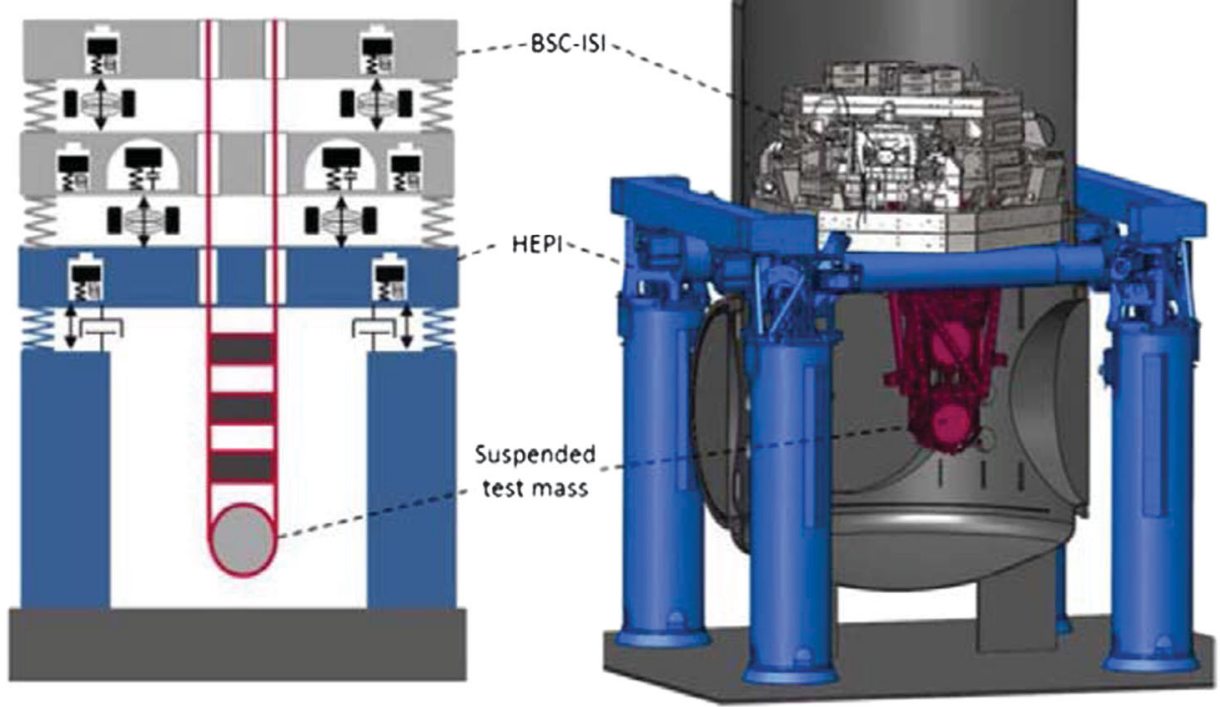

FIG. 5. Advanced LIGO multistage suspension system for the test masses with active-passive seismic isolation. 


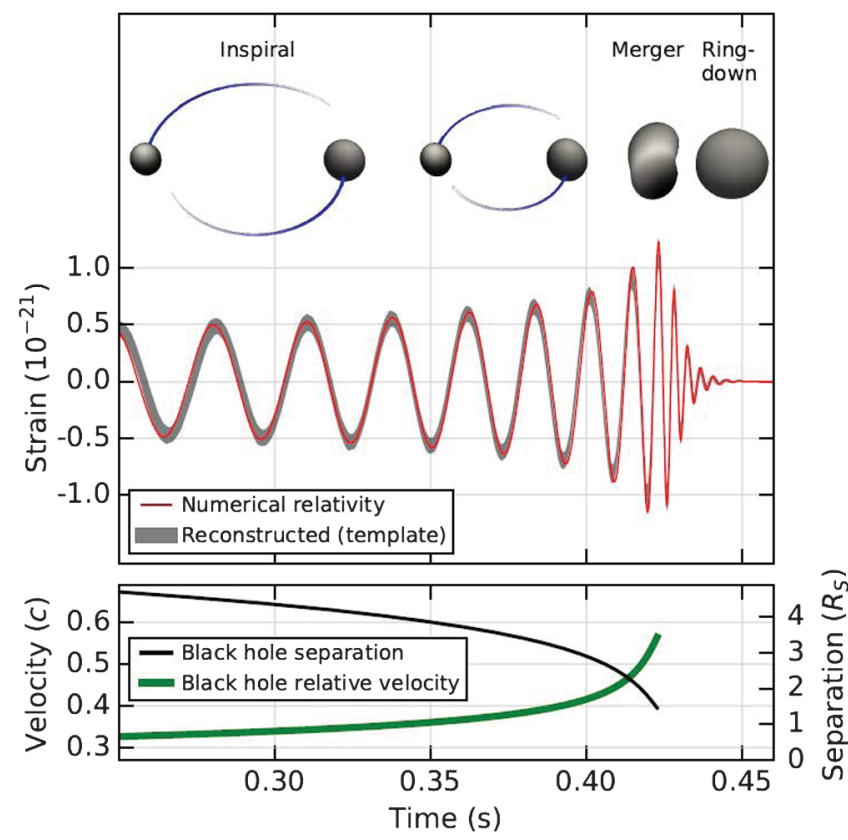

FIG. 6. The physics interpretation of the observed event as a binary black hole merger.

two heavy compact objects (black holes), each $\sim 30$ times the mass of the Sun, and going around each other separated by only a few hundred kilometers and moving at relativistic velocities.

On the top of the figure, we see the three different phases of the merger: the inspiral phase of the binary black holes system, then their merger of the two compact objects, and finally, there is a ringdown. The characteristic increasing frequency and amplitude with time (the so-called chirp signal) can be seen in the inspiral phase. The largest amplitude is during the final merger, and finally, there is a characteristic ringdown frequency. The two black holes inspiral and merge together due to the emission of gravitational radiation coming from the accelerations.

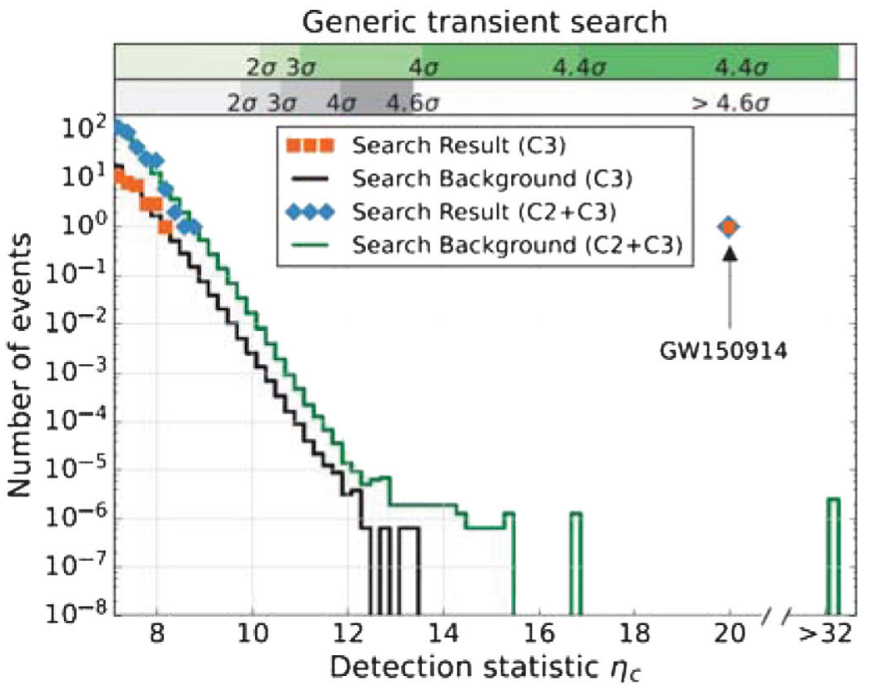

The bottom of Fig. 6 is even more revealing. The right-hand axis is basically in units of about $100 \mathrm{~km}$. That means the separation between the two merging objects at the beginning was only $\sim 400 \mathrm{~km}$, and at the end $\sim 100 \mathrm{~km}$. Therefore, these 30 solar mass objects are confined in a volume only about twice the size of Stockholm, yet the final black hole has 60 times the mass of our Sun, or $10 \times 10^{6}$ times as massive as the Earth. From the axis on the left, we see that when we first observed this event, the two objects were moving at about three-tenths the speed of light and that increases to over half the speed of light by the time of the final merges!

In order to be confident that what we observed was a real event and not a background fluctuation, we directly measure the background probability by comparing coincidence time slices for the two detectors, both in time (e.g. $\pm 10 \mathrm{~ms})$ and out of time! Since GW150914 occurred only days after we began data taking with Advanced LIGO, this required taking about one month of data before we could quantitatively establish the probability that the event was real. In other words, in addition to searching for coincidence in-time signals, we look for coincidences between all the out-of-time slices during our data taking. These background slices could not have come from any physical phenomena traveling at relativistic speeds, like gravitational waves. The total number of time slices we compared was equivalent to in-time background levels equivalent to over 67000 years of data taking. Taking into account the different event classes in our search, we reduce the limit on the false alarm rate to 1 in over 22500 years. This corresponds to a probability that our observed event is accidental to $<2 \times 10^{-6}$, establishing a significance level of $4.6 \sigma$. I emphasize that the measured significance level is set from the number of bins compared from 16 live days of data taking. This represents a lower limit on the actual significance of GW150914.

Figure 7 shows the statistical significance as described above for the GW150914 event, compared to the measured background levels, under two different assumptions. The horizontal axis is a measure of the significance of the events

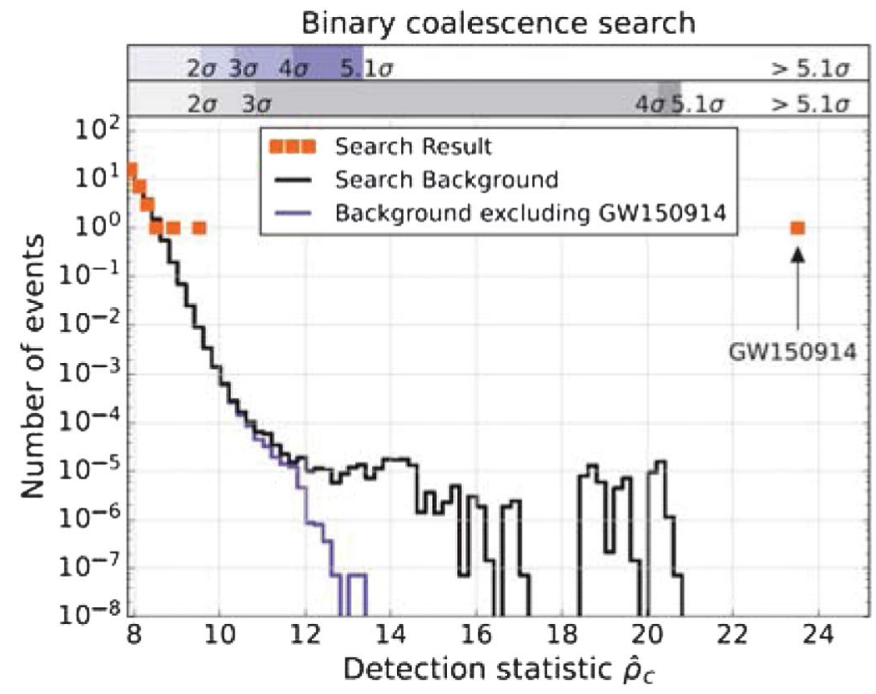

FIG. 7. The statistical significance of the event as a generic transient event on the left, and directly searching for events from a merger on the right. 
TABLE I. The main parameters of the black hole merger.

\begin{tabular}{lc}
\hline \hline Primary black hole mass & $36_{-4}^{+5} M_{\odot}$ \\
Secondary black hole mass & $29_{-4}^{+4} M_{\odot}$ \\
Final black hole mass & $62_{-4}^{+4} M_{\odot}$ \\
Final black hole spin & $0.67_{-0.07}^{+0.05}$ \\
Luminosity distance & $410_{-180}^{+160} \mathrm{Mpc}$ \\
Source redshift, $z$ & $0.09_{-0.04}^{+0.03}$ \\
\hline \hline
\end{tabular}

and the vertical access is the rate. The left-hand plot shows the observed GW150914 event at the level of one event level, and having statistical significance $\sigma>4.6$, as described above. This plot assumes a generic signal shape for the event. The right-hand plot shows a significance of $>5 \sigma$, when a binary coalescence form is assumed. Note that the second most significant event in these data is about $2 \sigma$, which may well also be a binary black hole merger, but at this early stage in LIGO, we are only declaring $5 \sigma$ events as gravitational wave binary mergers.

The shapes of the waveforms that describe the merger, coalescence and ringdown reveal the detailed parameters of the merger. The orbits decay as the two black holes accelerate around each other and emit energy into gravitational waves determined by the "chirp mass," as defined below, at leading order in the strength of the binary's gravity,

$$
\mathscr{M}=\frac{\left(m_{1} m_{2}\right)^{3 / 5}}{M^{1 / 5}} \simeq \frac{c^{3}}{G}\left[\frac{5}{96} \pi^{-8 / 3} f^{-11 / 3} \dot{f}\right]^{3 / 5} .
$$

The next order terms enable the measurement of the mass ratios and spins, the redshifted masses are directly measured, and the amplitude is inversely proportional to the luminosity distance. Orbital precession occurs when spins are misaligned with the orbital angular momentum. GW150914 shows no evidence for precession. The sky location is extracted from the time delay between detectors and the differences in the amplitude and phase in the detectors.

Using numerical simulations to fit for the black hole merger parameters, we determine that the total energy radiated into gravitational waves is $(3.0 \pm 0.5) M_{\odot} c^{2}$. The system reached a peak luminosity of $\sim 3.6 \times 10^{56} \mathrm{ergs} / \mathrm{s}$, and the spin of the final black hole $<0.7$ of the maximal black hole spin.

The main parameters of the black hole merger are summarized in Table I.

With only two detectors we cannot locate the direction very well, but comparing the time, amplitude and phase in the Livingston and Hanford interferometers, we are able to locate the gravitational wave as coming up from the Southern Hemisphere within an area of about 600 square degrees. Our most recent observations, discussed later, include Virgo and with three detectors we determine the sky location to tens of square degrees.

\section{MORE BLACK HOLE MERGERS}

The first Advanced LIGO data run (O1) continued for four months, from September 2015 to January 2016. Our second data run $(\mathrm{O} 2)$ ran from December 2016 to the end of August 2017. Much like the evolution described for Initial LIGO, we are making improvements between Advanced LIGO data runs and expect to achieve design sensitivity within a few years. We are actively searching for other signals, besides binary merges, including bust signals from phenomena such as supernova explosions or gamma ray bursts, continuous wave signals from spinning neutron stars (pulsars), stochastic background signals, etc. So far, we have only detected binary merger signals, but hope to detect others as our sensitivity improves.

We search for transient or burst events in two different ways. First, we use an unmodeled search for a power excess, using a wavelet technique, which makes no assumptions about the expected binary merger waveform. The second search method employs a matched template technique using binary merger waveforms. We have populated the available mass 1 vs mass 2 plane with templates that describe the waveform, calculated using post-Newtonian calculations and/or numerical relativity, as required. There are several hundred thousand templates, which are each multiplied by the observed noise in each interval of time (matched template technique).

Since announcing our observation of GW150914, we have reported (Abbott et al., 2016f, 2017a) several more observations of black hole mergers. As shown in Fig. 8, we have so far reported four black hole merger events having high statistical significance $(>5 \sigma)$, and one of $2 \sigma$, which we do not declare as an event. However, all the characteristics of that event are similar to the other black hole mergers, except that it is farther away, making the signal weaker and the signal to noise less significant.

It is interesting that we have only one such event candidate, having marginal signal to noise. This is a result of the extremely steep cutoff of the noise background when requiring a coincidence between the two sites, as is evident by the extremely sharp fall-off of the background noise in Fig. 7. We can conclude that there is very little correlation between the noisy events observed at the two sites. This was not necessarily anticipated, as we worried about correlated noise. However, this result is both more convincing, in terms of the events we have observed and bodes very well for the cleanliness of events in future observations by using the coincidence technique.

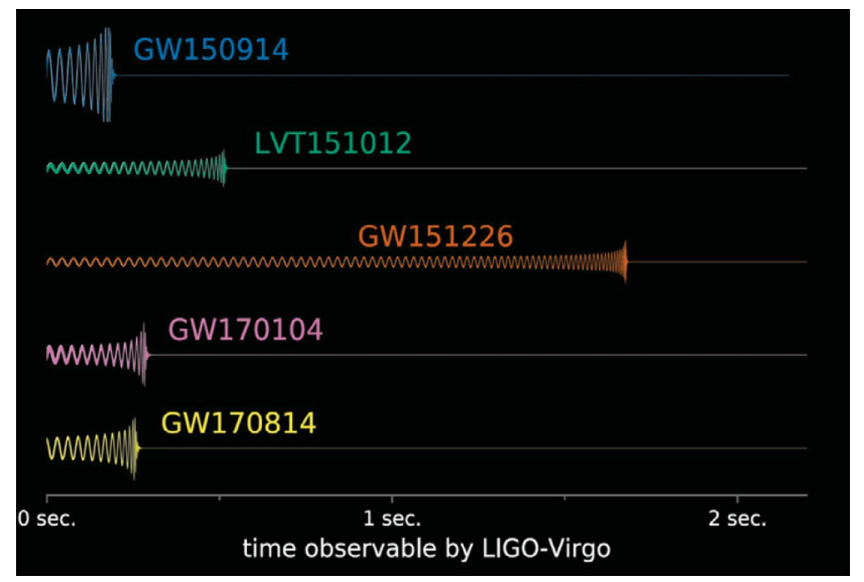

FIG. 8. Event characteristics of reported black hole mergers in LIGO. 
For the black hole merger events shown in Fig. 8, notice that the amount of time and number of cycles observable in LIGO is very dependent on the mass of the black hole system. The heaviest black hole we observed was the first one and we only observed a few cycles, while for the lightest one (GW151226) we have many cycles. Also, I repeat that the candidate event, LVT151-12, has characteristics completely consistent with the other events, but is about twice as far away and therefore has lower statistical significance.

\section{SCIENCE IMPLICATIONS OF THE OBSERVED BLACK HOLE MERGERS}

Gravitational waves represent a completely new way to view the Universe. We have every reason to expect that we will discover new phenomena and learn "new" astrophysics from gravitational waves. This has already been realized from the very first observations of gravitational events.

Conclusions from the first observations of black hole merger observations from gravitational waves include the following:

- Stellar binary black holes exist.

- They form into binary pairs.

- They merge within the lifetime of the Universe.

- Masses $\left(M>20 M_{\odot}\right)$ are considerably heavier than what was known or expected of stellar mass black holes.

The fact that the observed black hole mergers in LIGO are so massive has opened the question of how they were produced. LIGO is strongly biased to detecting heavy masses, because the stronger signal provides sensitivity to greater distance and the volume observed grows like the cube of the distance. Nevertheless, the creation of such large masses needs explanation. If these stellar mass black holes come from the gravitational collapse of heavy stars, it requires special conditions for the parent star to survive, such as low-metallicity regions of the Universe. Another possibility is that the heavy black holes were produced in dense clusters, and a third is that they are primordial and may even be associated with the dark matter; see Fig. 9.

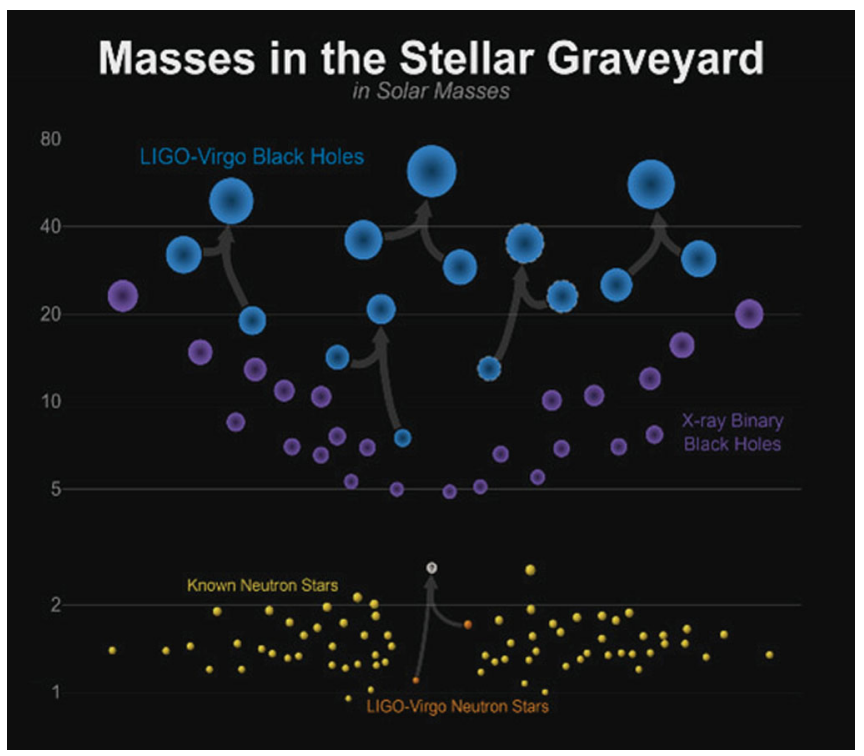

FIG. 9. Masses of observed black hole mergers in LIGO.

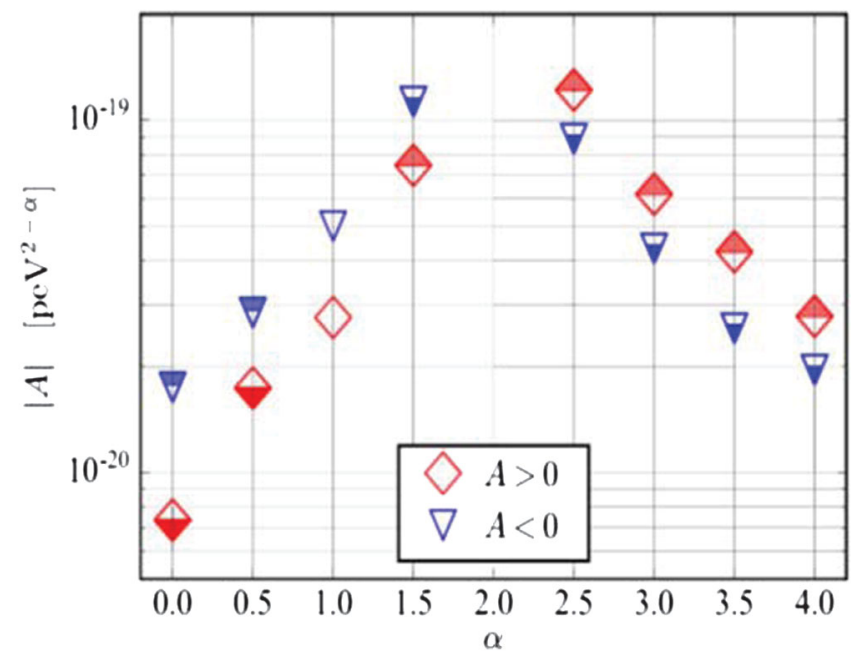

FIG. 10. Tests of the inclusion of a dispersion term in general relativity. Upper limits are shown on the presence of a dispersion term.

The next challenge will be to distinguish between these or other possibilities for the origin of such heavy stellar black holes. More events will give us distributions of masses and other parameters, while larger signal/noise events will enable determining the other feature of these mergers. For example, are the spins of the merging black holes aligned, antialigned or is there no correlation in the spins?

Our other major scientific goal is to test general relativity in the important regime of strong field gravity. The merger of black holes presents just such a new "laboratory" for these studies. We can compare the observed waveform with the predictions of general relativity looking for deviations. So far, all tests are in good agreement with the theory.

The first test is just how well general relativity fits the details of our data, or if there are any other features in the data. At the present level, the deviations we see are all consistent with the instrumental noise in our interferometers.

We have tried to quantify our level of agreement by adding a dispersion term, which would indicate that some components might not travel at the speed of light and are dispersed. The large distance to the black hole merger events provides good sensitivity for such tests. From Fig. 10 we see that very little dispersion is allowed. The limit $\alpha=0$ corresponds to an upper limit on the mass of gravitons (a hypothetical particle mediating the force of gravity). Assuming that gravitons are dispersed in vacuum like massive particles, then the bound on graviton mass is $M_{g}<7.7 \times 10^{-23} \mathrm{eV} / c^{2}$

More stringent tests of general relativity will be possible as we collect more events, and in particular we can test alternate formulations of general relativity.

\section{VIRGO DETECTS GRAVITATIONAL WAVES}

Finally, very recently we reported (Abbott et al., 2017b) one more black hole merger event (GW170814) and for the first time this was also observed in the Virgo detector near Pisa in Italy (see Fig. 11). Virgo is a collaboration of France, Italy, Netherlands, Poland and Hungary. The founders of Virgo were 


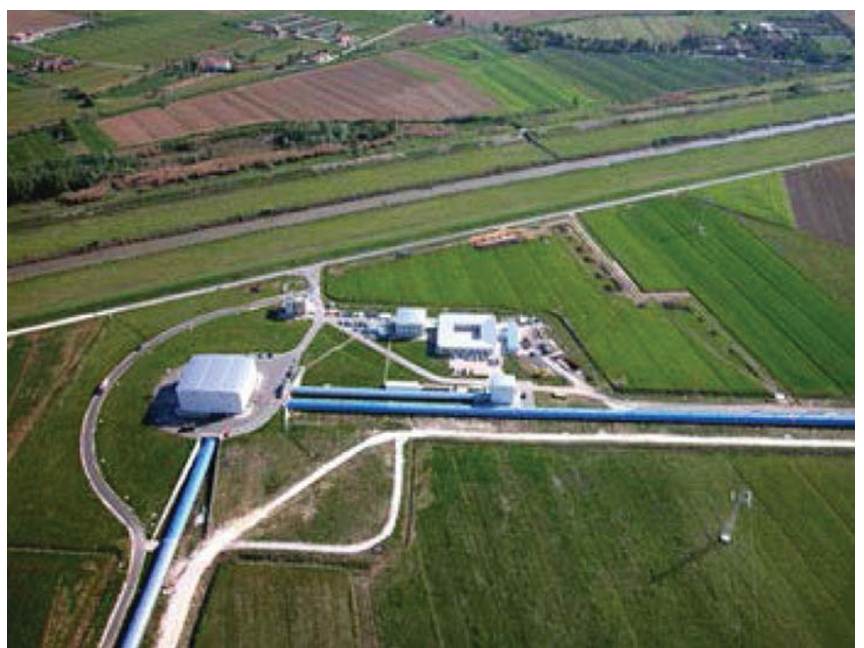

FIG. 11. The Virgo Suspended Mass Interferometer in Cascina, Italy.

Adalberto Giazotto and Alain Brillet and the present leadership is Federico Ferrini as the European Gravitational Observatory (EGO) director and Jo van de Brand as Virgo spokesperson. Virgo is a $3 \mathrm{~km}$ interferometer, similar to LIGO, but with some technical differences.

Adding Virgo, in this detection, not only gives independent confirmation of the LIGO black hole merger detections, but improves markedly the ability to triangulate. This is a precursor to also adding KAGRA in Japan and LIGO-India detectors to the network. The combination will give good sky coverage and more than an order-of-magnitude improvement in locating the direction of the source. This can be seen in Fig. 12, where the sky location of this last observed event is dramatically better than the previous observed black hole merger events.

The first three-way coincidence enabled a test of general relativity that could not be carried out with LIGO alone, because the two LIGO detectors are almost coaligned. Including the nonaligned Virgo data, the polarization of the gravitational waves can be studied to determine whether the waves are transverse as predicted in general relativity. GW170814 enabled the first tests for nontransverse polarizations. The first such analysis was performed using polarizations that are forbidden by general relativity and these were disfavored in the analysis. More precise tests and analysis will be possible in the future using events detected in both LIGO and Virgo.

\section{NEUTRON STAR BINARY MERGER}

Two weeks after the Nobel announcement in October and almost two months before this lecture, we announced the first observation of a merger of a neutron star binary system (see Fig. 13) (Abbott et al., 2017c). This was also the first gravitational wave event to have electromagnetic counterparts observed in a large variety of astronomical instruments (see Fig. 15). This event initiates the eagerly sought after new field of multimessenger astronomy.

On 17 August 2017, LIGO and Virgo registered a gravitational wave signal from the inspiral of two neutron stars. Many of us expected the first source we would observe in LIGO would be from the merger of binary neutron stars, because there is a known rate of detected binary neutron stars in our own galaxies by radio telescopes. Although accurately predicting the rate of mergers detectable by LIGO from these data is not very precise, we knew it to be in the range of detectability by LIGO. No such definite predictions were possible for binary black hole mergers or for black holeneutron star mergers (which we have not yet detected).

Neutron stars are very dense nuclear matter and one of the main goals studying such describe superdense matter by a relationship called the "equation of state." The neutron stars mass and equation of state determines size and tidal deformation from the gravitational pull of the companion neutron star. We cannot determine the equation of state from this event, but this is a future goal from these detections.

From our estimation of the parameters, the masses of the two compact objects turn out to be between 0.86 and 2.26 solar masses as shown in Fig. 14. In fact, restricting the spins to the range for binary neutron stars limits the mass
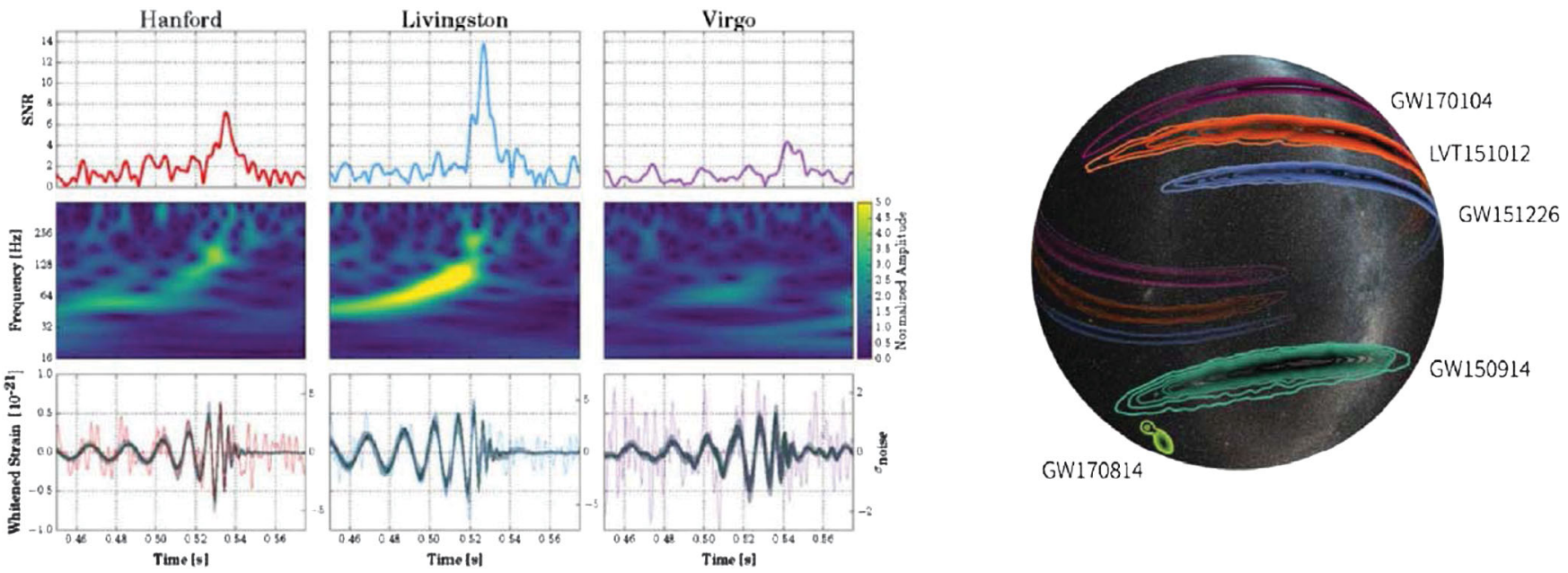

FIG. 12. Black hole merger GW170814 showing LIGO-Hanford, LIGO-Livingston and Virgo signals on the left, and the reconstructed direction of the gravitational wave on the right. 


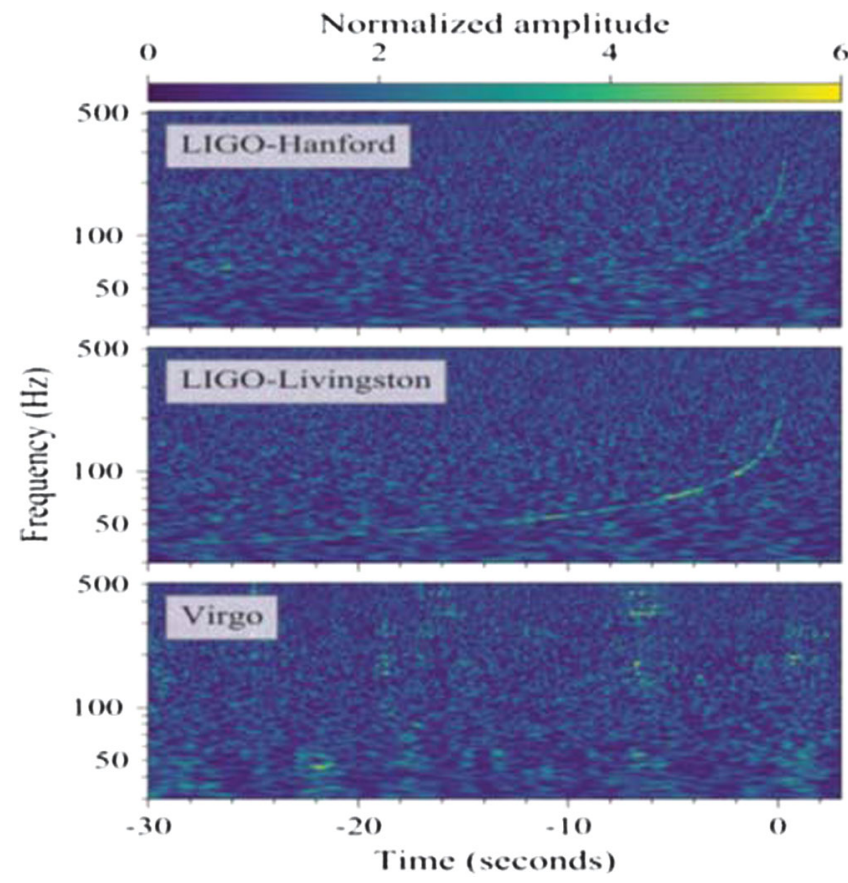

FIG. 13. The first observation of binary neutron star merger. Time-frequency representations of gravitational wave event GW170817, observed by the LIGO-Hanford (top), LIGO-Livingston (middle), and Virgo (bottom) detectors.

determination to between 1.17 and 1.60 solar masses, consistent with being neutron stars. The distance to the source is well determined from the amplitude of the merger gravitational wave signal to be about $40 \mathrm{Mpc}\left(130 \times 10^{6}\right.$ light years $)$.

This event occurred only a few days after Virgo had joined the gravitational wave network and greatly improved localization for the black hole merger event discussed above. The localization of the neutron merger event, including Virgo, is an oblong about 2 deg across and 15 deg long, covering about 28 square deg.

The Fermi satellite observed a gamma ray burst GRB170817A in the same region of the sky and the triggered follow-ups identified the fading light from the event from near NGC 4993, first by the $1 \mathrm{~m}$ Swope optical telescope
(LIGO et al., 2017). The gravitational wave observation of the neutron star merger provides a new independent method of measuring the Hubble constant, the rate of expansion of the Universe, using a method proposed by Bernard Schutz, called a "standard siren" measurement (see Fig. 16) (Schutz, 1986). The idea is to use the distances of galaxies determined just from the gravitational-wave observations, by determining the luminosity (e.g. distance) directly from the observations. Standard sirens are compact binary systems, consisting of neutron stars or black holes, whose gravitational waveform as the compact objects inspiral toward merger carries information about the distance of the source, as well as the masses of the compact objects and other parameters of the system. In this case, we use the precise information of which galaxy the event occurred in from the optical observations.

GW170817 represents the first joint detection of gravitational and electromagnetic waves from the same astrophysical source. All the data agree with the hypothesis that the source is the merger of two neutron stars in the host galaxy NGC4993 in the constellation of Hydra. Analysis of the waveform of GW170817 yielded a distance estimate of about $44 \mathrm{Mpc}$, assuming that the sky position of GW170817 was exactly coincident with its optical counterpart. The gravitational wave distance estimate is completely independent of the cosmic distance ladder derived from electromagnetic observations, so that future measurements with more statistics from gravitational waves can help distinguish the uncertainties between the electromagnetic observations.

The observation in LIGO and companion observation of a short gamma ray burst opened a full astronomical campaign to observe this event in different wavelengths and devices, including the large neutrino detectors (see Fig. 17). I will not summarize those results here (Abbott et al., 2017d, 2017e, 2017f), as that is not the subject of this Nobel Lecture, but the rich observations gave strong support to the "kilonova" picture of a binary neutron star merger, as well as that neutron star binary mergers are a significant source of the heavy elements in nature. Even more exciting is the fact that the long anticipated idea of doing multimessenger astronomy-using the complementary information from electromagnetic, neutrinos and gravitational waves to study the same phenomenahas become a reality.
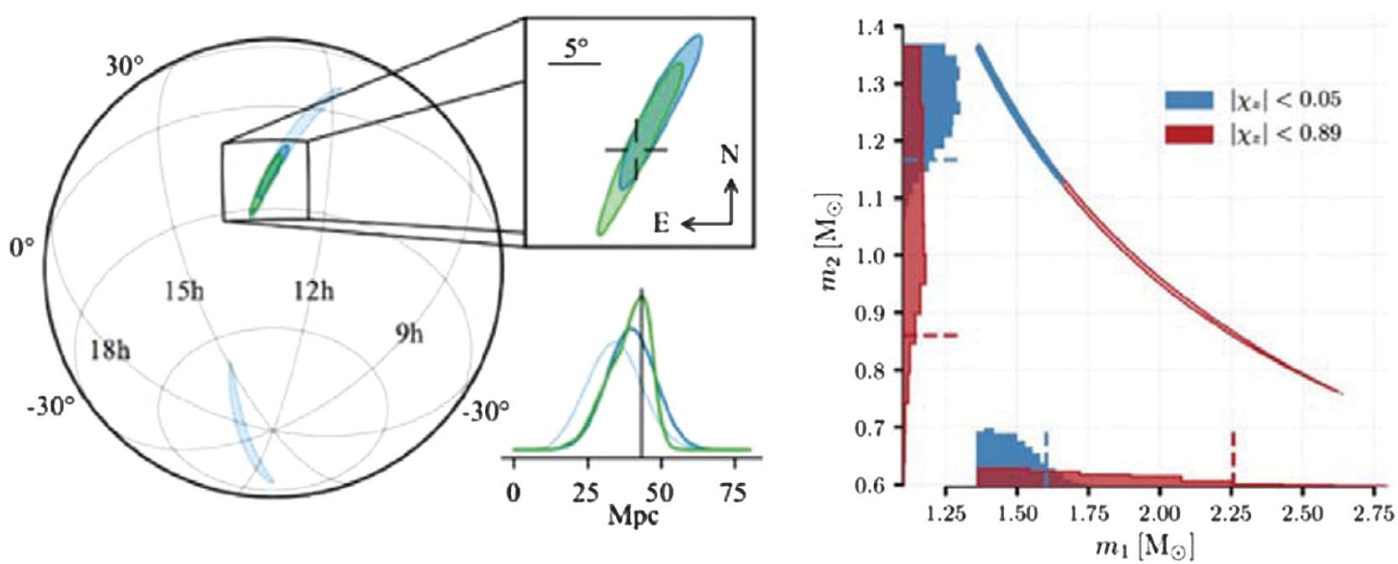

FIG. 14. The main parameters of GW170817. 

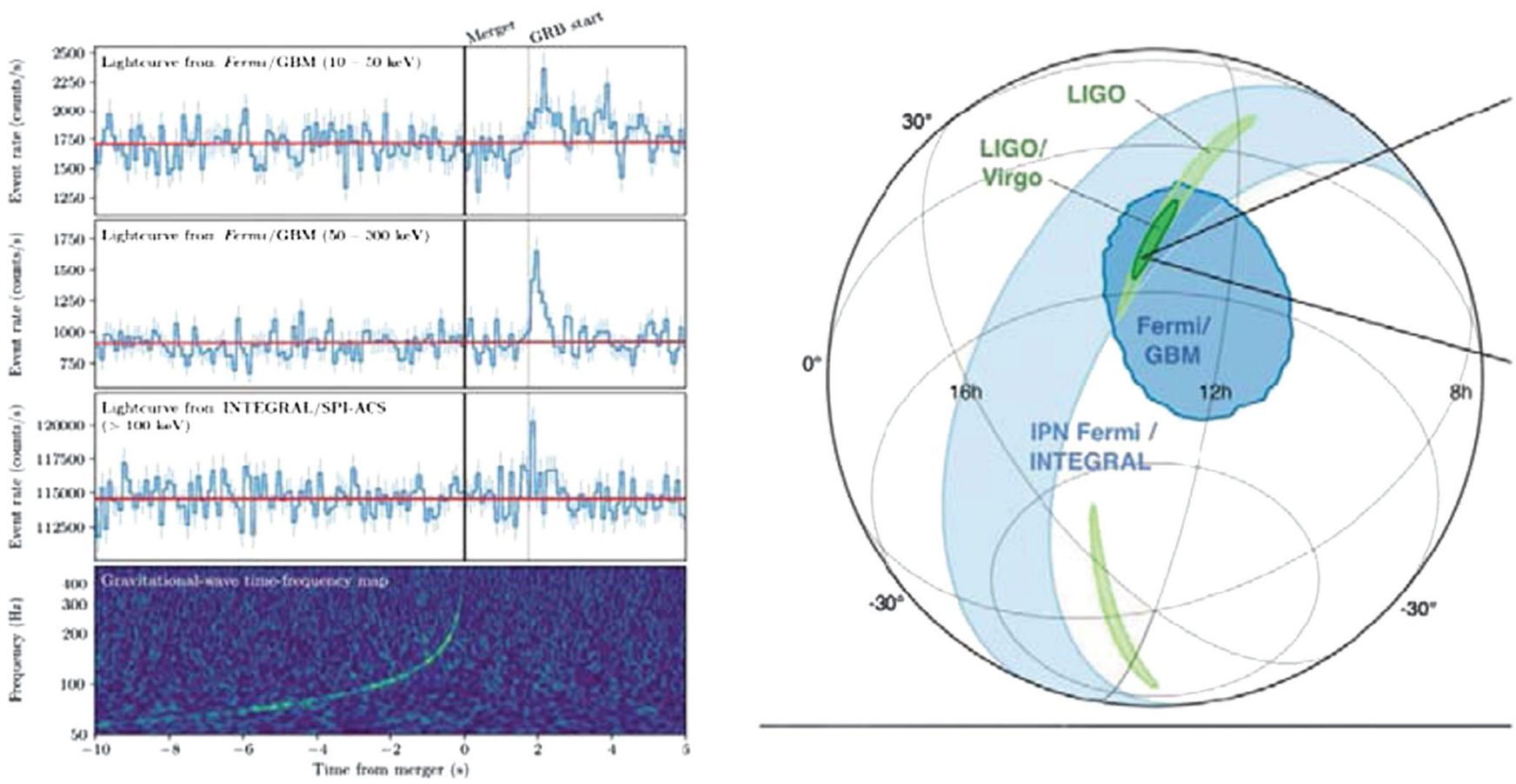

FIG. 15. Fermi short gamma ray burst signal observed $1.7 \mathrm{~s}$ after the gravitational wave signal in the same region of the sky.

\section{FUTURE DIRECTIONS FOR LIGO AND GRAVITATIONAL WAVES}

As discussed above, we have completed two data taking runs (O1 and O2) with Advanced LIGO. In the first data run, we made the first direct observation of gravitational waves, resulting from a black hole merger $\sim 1.3 \times 10^{9}$ years ago. The waveform data fit the predictions of Einstein's general relativity remarkably well and all detailed analyses are in good agreement with general relativity. The merging black holes were each about 30 solar masses, which is surprisingly heavy. During the same data run, we observed a second black hole merger about three months later, again having more than $5 \sigma$ significance. During this data run, we also observed a weaker event, having about $2 \sigma$ significance, which we do not claim as a gravitational wave event, but again appears to fit the black

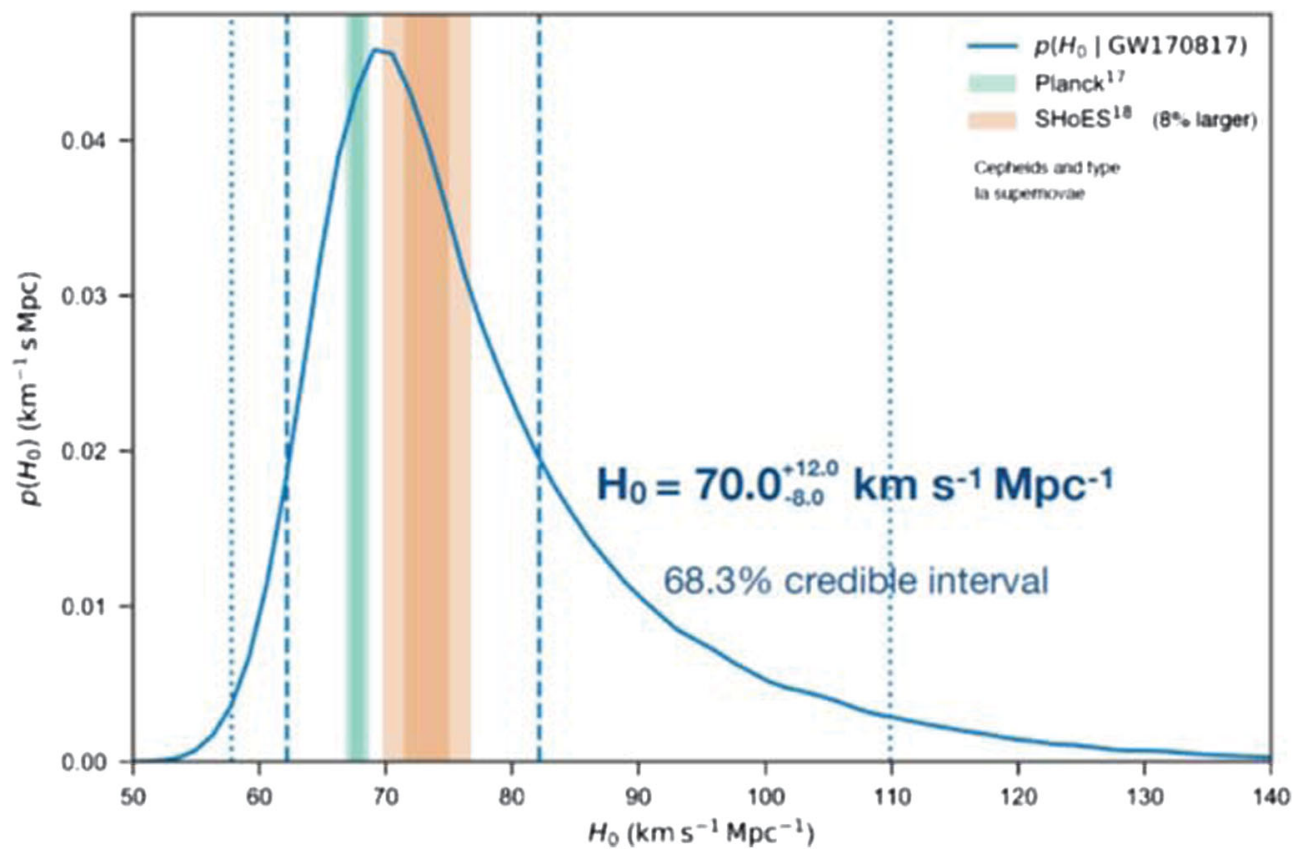

FIG. 16. Determination of the Hubble constant from gravitational wave data. The blue curve shows the relative probability of different values of $H_{0}$ with the peak at $70 \mathrm{~km} \mathrm{~s}^{-1} \mathrm{Mpc}^{-1}$. The dashed and dotted blue vertical lines are the limits of $68.3 \%$ and $95.4 \% \mathrm{credible}$ intervals for $H_{0}$. Our gravitational wave results are in agreement with both Planck and SHoES. Note that we cannot distinguish from just one event between these two, which disagree with each other at the $95.4 \%$ probability level. 


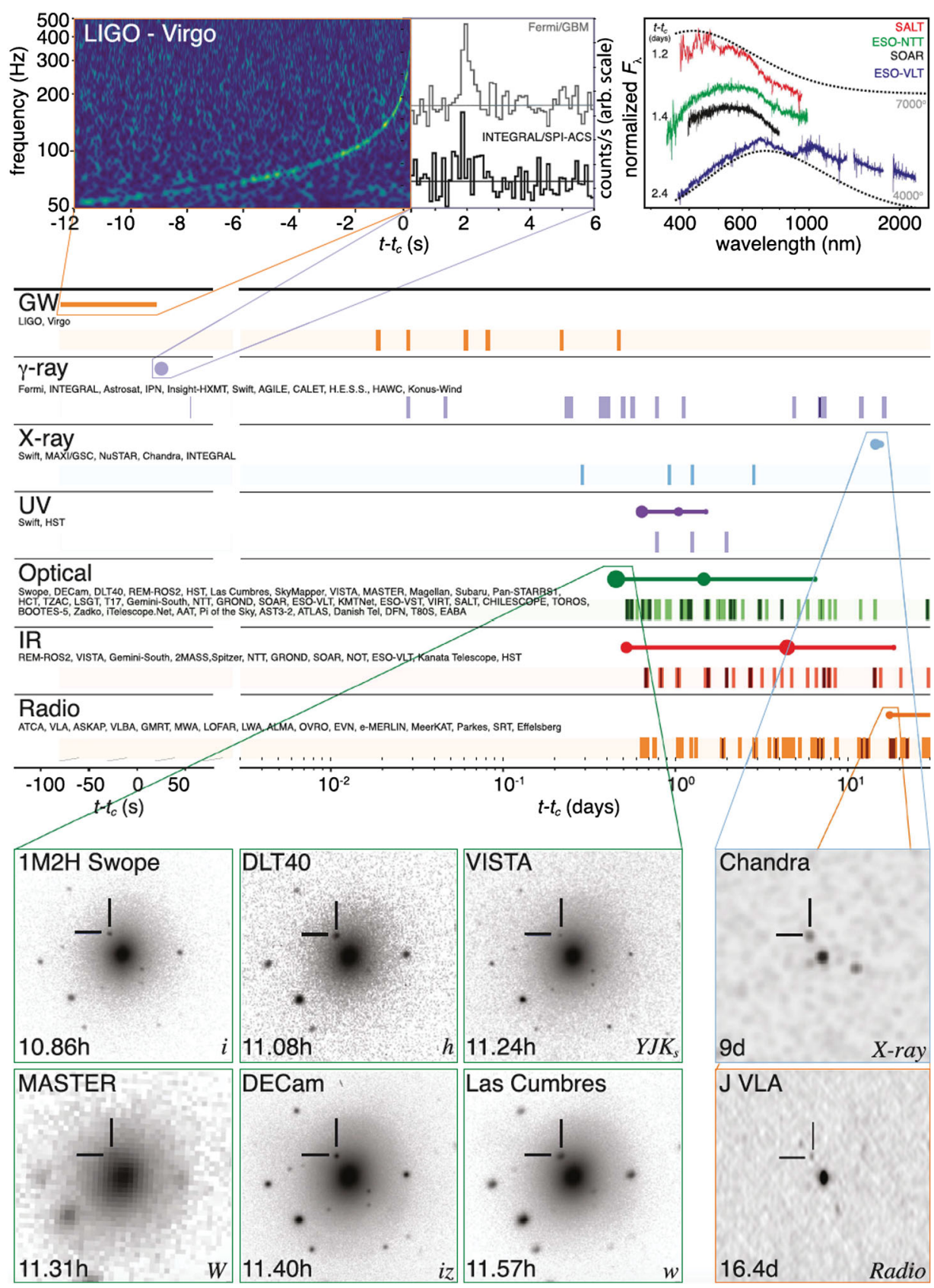

FIG. 17. The electromagnetic campaign for counterparts to GW170817.

hole merger hypothesis very well; however this occurred about twice as far away as the other two events and therefore has a weaker signal and is less significant.

Following O1, we spent about nine months calibrating the interferometers and improving them. The sensitivity improvements for $\mathrm{O} 2$ were within the range of our planning, but technical problems, especially in Hanford, limited the improvements. We could hardly expect $\mathrm{O} 2$ to be as spectacular as $\mathrm{O} 1$, especially since our sensitivity improvements were less than we had hoped for. However, $\mathrm{O} 2$ has turned out to be comparably exciting to $\mathrm{O} 1$. We recorded several more black hole mergers and are starting to get enough to get some statistical information on mass distributions, etc. Near the end of the data taking, in August 2017, the Virgo detector in Italy had good enough sensitivity to join LIGO as part of a network. A short time later, we observed another black hole merger, but this time with a threefold coincidence. As expected, the third detector dramatically improved our ability to locate the source on the sky from many hundreds of square degrees to a few tens of square degrees. This demonstrated our future ability to compare information with other astronomical instruments looking for signals from the same phenomena. No companion electromagnetic signals are expected for black hole mergers and none have been seen.

Finally, as discussed above, we have made the first observation of gravitational waves from a neutron star merger. Using the combined LIGO and Virgo observations produced a much better source location, enabling all the related electromagnetic observations discussed above. This was a spectacular initiation of multimessenger astronomy and portents exciting future combined observations. 


\section{THE FUTURE}

We are now in the process of preparing for our third observational run, $\mathrm{O} 3$, which will begin in about $1 \mathrm{yr}$. The long down periods for LIGO are a direct result of the large premium in improving the interferometer sensitivities. We are presently about a factor of 2 away from Advanced LIGO design sensitivity. Figure 18 shows the performance improvement planned for Advanced LIGO and what was achieved for the $\mathrm{O} 1$ observing run. We made some modest improvements for $\mathrm{O} 2$ and expect to make more for $\mathrm{O} 3$, the next data run. In steps, we expect to reach the design sensitivity over the next few years.

The worldwide gravitational wave network will add the KAGRA interferometer in Japan and the LIGO-India interferometer over the next decade, giving both improved location information and broad sky coverage.

The rates for the black hole and neutron star mergers by more than an order of magnitude, compared to $\mathrm{O} 1$ and $\mathrm{O} 2$. In addition to compact binary mergers, over the next decade, we will be able to study the features and science of compact binary mergers, and it is possible that the improved sensitivity will open up other gravitational wave channels to observations.

In addition, we are working on a set of improvements beyond Advanced LIGO (Miller et al., 2015) that are to be implemented over the next decade. We call this improvement program $\mathrm{A}+$, and we believe it is a realistic goal to do the enabling R\&D and implement improvements over the coming decade that will improve the sensitivity by another factor of 2 beyond advanced LIGO. We are preparing to submit a specific proposal to the NSF in the near future.

In addition, we are working on technological improvements to fully exploit the potential of the present LIGO facilities and sites (Voyager), as well as investigating concepts for a third generation detector (Cosmic Explorer). Figure 18 shows our present view of improvements that could ultimately be made fully exploiting the present LIGO sites. The R\&D to develop Voyager could be incorporated as such a third generation

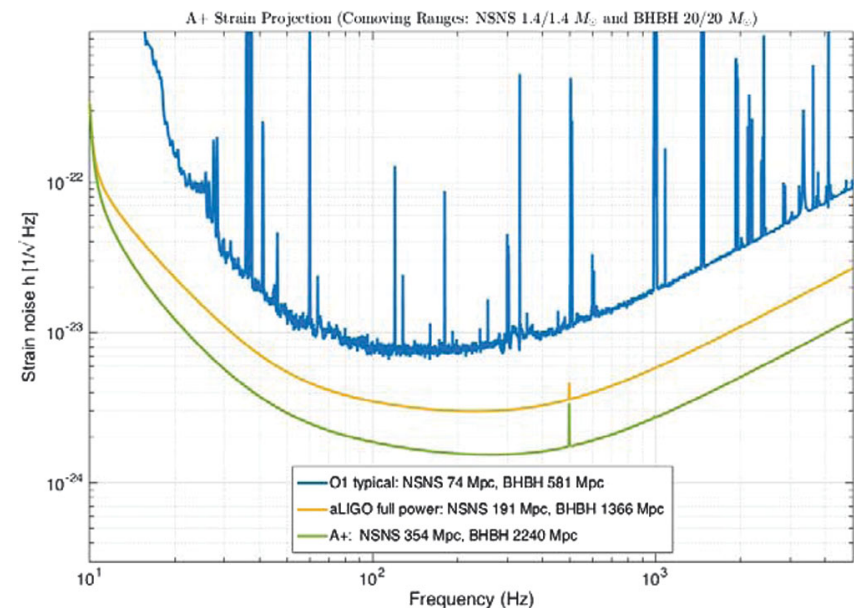

FIG. 18. Typical LIGO sensitivity during the O1 data run, the Advanced LIGO design sensitivity, and the estimated sensitivity achievable with the A+ upgrades. A+ will involve a variety of improvements over the present LIGO, including implementing squeezed light, improved optical coatings and test masses, and possibly cryogenic cooling. detector. It appears possible to improve the sensitivity of LIGO by about a factor of 10 beyond what has presently been achieved.

The past two years have seen an impressive beginning to this new field, based on gravitational wave observations. We hope to make a set of incremental improvements over the next decade, first achieving the Advanced LIGO and, hopefully, followed by continued improvements to fully exploit the sites (see Fig. 19), achieving x10 the present sensitivity.

This will be far from the end of the story. Conceptual studies for the Cosmic Explorer (McClelland et al., 2015) in the U.S., and especially for the Einstein Telescope (Punturo and Somiya, 2013) in Europe illustrate that we should be able to make third generation ground based detectors that will be 10 times more sensitive than can be achieved at the present LIGO facilities. The Einstein Telescope is designed to have sensitivity $\mathrm{x} 10$ better than LIGO. The key features in the concept are that it is deep underground, has $10 \mathrm{~km}$ arms and a triangular configuration, is cryogenic and has both a low frequency optimized and a high frequency optimized configuration. The Cosmic Explorer concept is less mature at this time, but is basically a 10x scale-up of LIGO and on the surface. Which is the better approach, how many detectors are needed in the next generation for pointing are unresolved. The community is now doing a study of the science potential for a new generation of detectors and this will help set the priorities in developing a unified concept to pursue.

It is clear that it is possible to make future detectors that will open up impressive new science possibilities with gravitational waves as discussed in Kip Thorne's lecture. He also discusses the future science for LISA, the planned spacebased gravitational wave experiment, as well as pulsar timing and other possible future gravitational wave probes.

We eagerly look forward to a deeper understanding of our Universe and likely, many exciting surprises, as we open and exploit this new field of gravitational wave science.

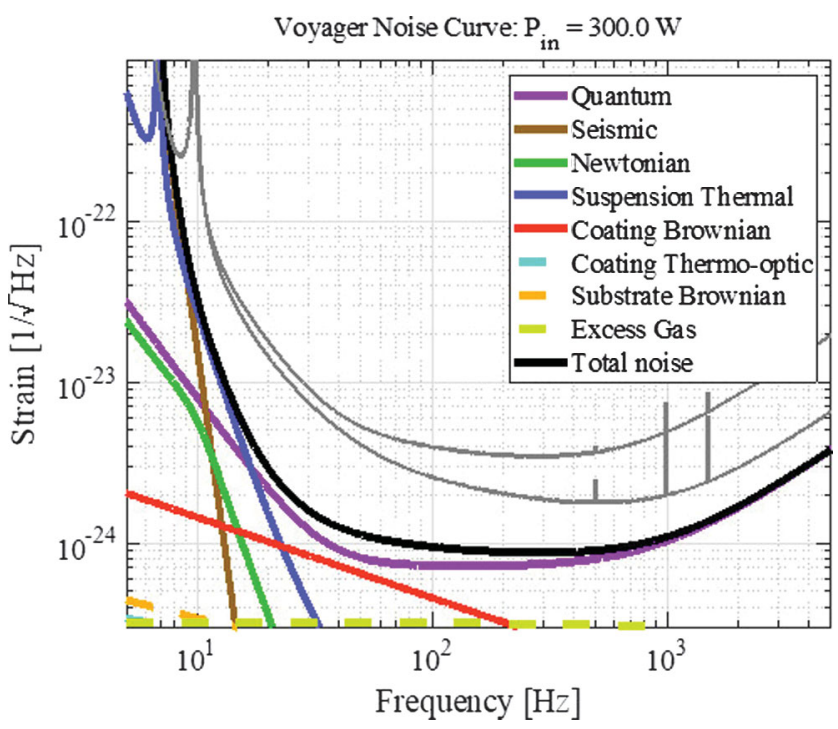

FIG. 19. Fully exploiting the LIGO sites. Employing improved technologies, a factor of x10 improvement over O1 sensitivities appears possible. 


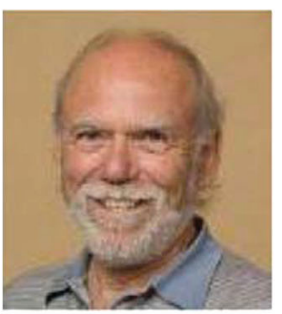

Barry Barish

Former Director
LIGO Laboratory Directorate

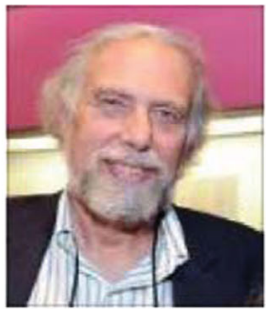

Jay Marx

Former Director

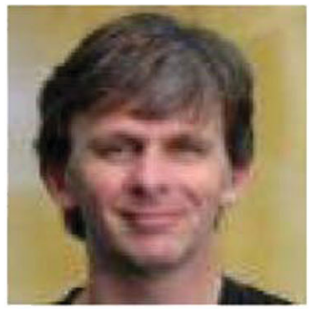

Dave Reitze

Director

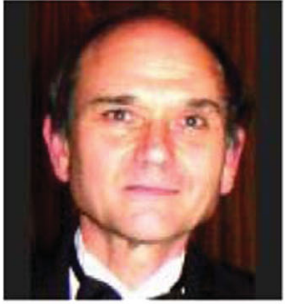

Albert Lazzarini

Deputy Director

FIG. 20. LIGO Laboratory Directorate members including Barry Barish, Jay Marx, Dave Reitze, and Albert Lazzarini.

\section{LIGO Scientific Collaboration Spokespersons}

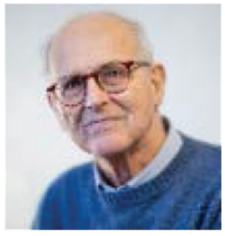

Rai Weiss

Former

Spokesperson

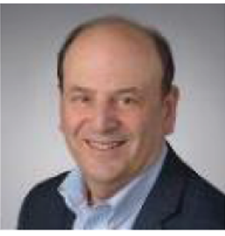

Peter Saulson

Former

Spokesperson

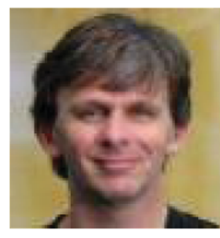

Dave Reitze

Former

Spokesperson

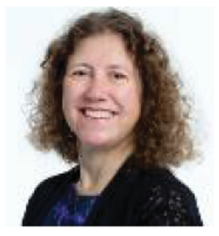

Former

Spokesperson

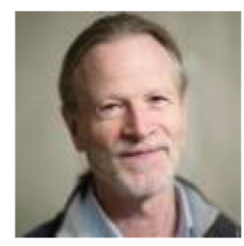

Shoemake

Spokesperson

FIG. 21. LIGO Scientific Collaboration Spokespersons including Rai Weiss, Peter Saulson, Dave Reitze, Gabriela Gonzalez, and David Shoemaker.

\section{FINAL REMARKS AND REFLECTIONS}

LIGO has been a remarkable adventure, beginning with Einstein's prediction in 1916, and some later wavering in his own belief in gravitational waves. There was a long road, bringing us into the 1950 s before the theoretical community became fully convinced of gravitational waves. The experimental part of the story started in earnest in the 1950s when Joseph Weber began the first serious attempts to detect gravitational waves with resonant bars. Some of the early history was marred by false discoveries, but overall, much was learned from the resonant bar era that has been built into the way we approach using interferometers. The concept of doing coincidences from separated detectors to be confident of detections, the analysis of background noise sources is used extensively in LIGO, and finally analyzing off-coincident time slices to measure accidental background levels.

The early interferometer era is covered in Rai Weiss's lecture. It is characterized by a broad worldwide R\&D effort, incorporating ideas and testing them in large scale prototypes. Most of the key concepts employed in Advanced LIGO were first suggested and tested several decades ago.

LIGO, itself, has been built and operated by a very strong collaboration between the Caltech and MIT groups, with some key contributions from collaborators in Scotland, Germany, Australia and several U.S. universities. The LIGO Scientific Collaboration has carried out the data analysis and written our series of discovery papers in a way that we can all be proud.

Finally, our home institutions, Caltech and MIT, have given us unusual support and encouragement through all the years

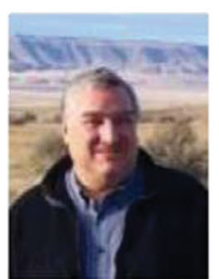

Fred Raab

Former Hanford

Head

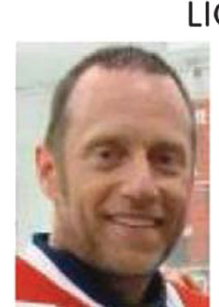

Mike Landry

Hanford

Head

LIGO Site Heads

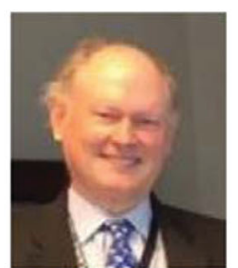

Mark Coles

Former

Livingston

Head

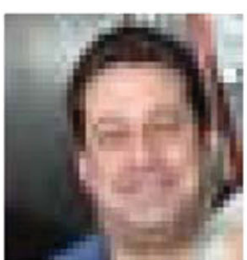

Mike Zucker

Former

Livingston

Head

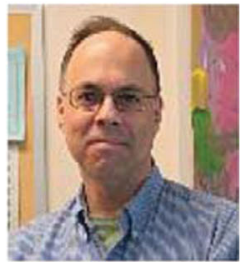

Joe Giaime

Livingston

Head

FIG. 22. LIGO Site Heads including Fred Raab, Mike Landry, Mark Coles, Mike Zucker, and Joe Giaime. 


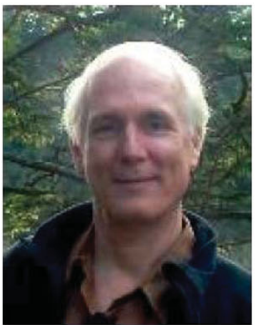

Dennis Coyne Chief Engineer

Advanced LIGO

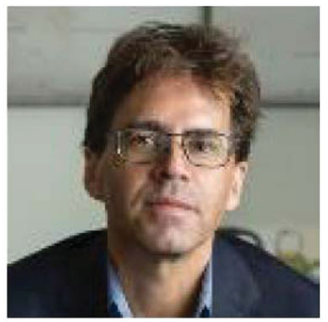

Peter Fritschel Interferometer Design

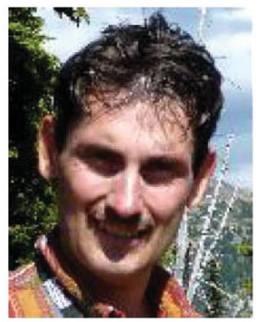

Daniel Sigg

Interferometer

Operations

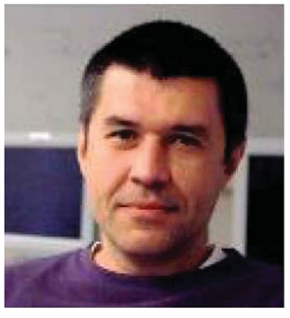

Valery Frolav

Interferometer

Operations

FIG. 23. Advanced LIGO members including Dennis Coyne, Peter Fritschel, Daniel Sigg, and Valery Frolav.

LIGO Lab - Senior Scientists

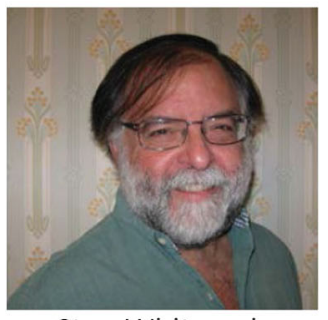

Stan Whitcomb

Chief Scientist

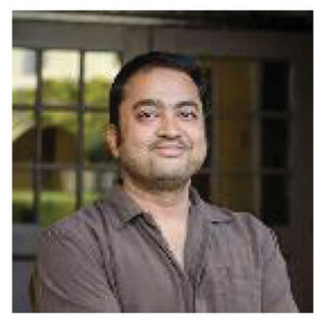

Rana Adakari

Future Detectors

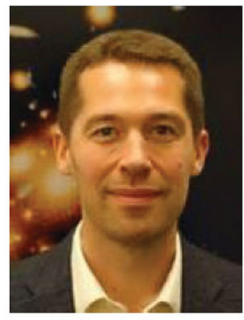

Matt Evans Future Detectors

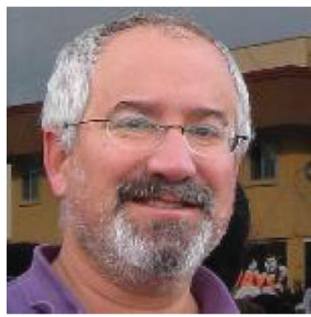

AlanWeinstein

Open Data

FIG. 24. LIGO Lab, Senior Scientists, including Stan Whitcomb, Rana Adakari, Matt Evans, and Alan Weinstein.

we toiled, before achieving the ultimate success, detections of gravitational waves. But, most of all, we are indebted to the U.S. National Science Foundation, for being an amazing institution, where supporting and facilitating the best science is what they do. Thank you, all!

\section{LIGO-SOME KEY MEMBERS}

We are especially indebted to the LIGO Laboratory, who performed the enabling $\mathrm{R} \& \mathrm{D}$, the interferometer designs, the construction of LIGO, the commissioning, the operations and upgrades. The LIGO Laboratory has worked closely with the 1200 member LIGO Scientific Collaboration, who have been responsible for the data analysis and science. By necessity, the key members of the LIGO Lab shown below, represent only a partial list; see Figs. 20-24.

\section{ACKNOWLEDGMENTS}

I would especially like to acknowledge the U.S. National Science Foundation for their very long-term commitment and support of LIGO.

\section{REFERENCES}

Aasi, J., et al., 2013, Phys. Rev. D 87, 022002. Abbott, B. P., et al., 2009, Rep. Prog. Phys. 72, 076901. Abbott, B. P., et al., 2016a, Phys. Rev. Lett. 116, 061102. Abbott, B. P., et al., 2016b, Phys. Rev. Lett. 116, 131103. Abbott, B. P., et al., 2016c, Phys. Rev. D 93, 122003. Abbott, B. P., et al., 2016d, Phys. Rev. Lett. 116, 221101.
Abbott, B. P., et al., 2016e, Astrophys. J. Lett. 833, L1.

Abbott, B. P., et al., 2016f, Phys. Rev. Lett. 116, 241103.

Abbott, B. P., et al., 2017a, Phys. Rev. Lett. 118, 221101.

Abbott, B. P., et al., 2017b, Phys. Rev. Lett. 119, 141101.

Abbott, B. P., et al., 2017c, Phys. Rev. Lett. 119, 161101.

Abbott, B. P., et al., LIGO and VIRGO Collaborations, 2017d, Astrophys. J. Lett. 850, L39.

Abbott, B. P., et al., LIGO, Virgo, Fermi, and Integral Collaborations, 2017e, Astrophys. J. Lett. 848, L13.

Abbott, B. P., et al., 2017f, Astrophys. J. Lett. 848, L12.

Abramovici, et al., 1996, Phys. Lett. A 218, 157-163.

Advanced LIGO, 2015, Classical Quantum Gravity 32, 074001.

Einstein, A., 1916, Sitzungsber. K. Preuss. Akad. Wiss. 1, 688.

Einstein, A., 1918, Sitzungsber. K. Preuss. Akad. Wiss. 1, 154.

McClelland, David, Matthew Evans, Brian Lantz, Ian Martin, Volker Quetschke, and Roman Schnabel (LIGO Scientific Collaboration), 2015, Instrument Science White Paper (PDF) (Report), LIGO Document T1500290-v2.

Miller, John, Lisa Barsotti, Salvatore Vitale, Peter Fritschel, Matthew Evans, and Daniel Sigg, 2015, Phys. Rev. D 91, 062005.

Punturo, M., and K. Somiya, 2013, Int. J. Mod. Phys. D 22, 1330010.

Riordan, M., L. Hoddeson, and A. Kolb. 2015. Tunnel Visions: The Rise and Fall of the Superconducting Super Collider. (University of Chicago Press, Chicago).

Schutz, B. F., 1986, Nature (London) 323, 310.

Shoemaker, D., et al., 1988, Phys. Rev. D 38, 423.

The LIGO Scientific Collaboration and The Virgo Collaboration, The 1M2H Collaboration, The Dark Energy Camera GW-EM Collaboration and The DES Collaboration, The DLT40 Collaboration, The Las Cumbres Observatory Collaboration, The VINROUGE Collaboration, and The MASTER Collaboration, 2017, Nature (London) 551, 85. 